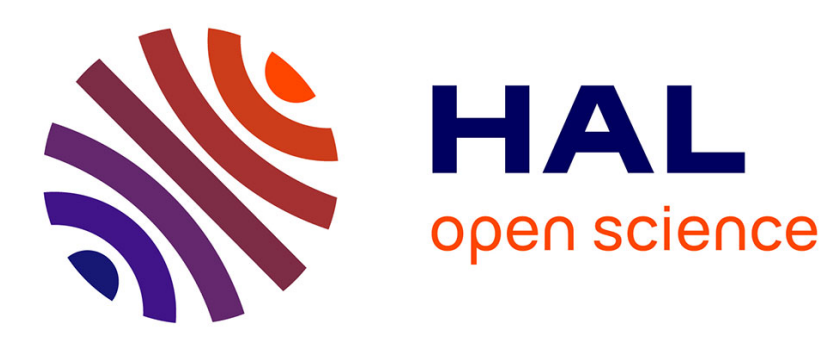

\title{
Thin wedge evaporation/condensation controlled by the vapor dynamics in the atmosphere
}

\author{
F. Doumenc, V. Janeček, V. S. Nikolayev
}

\section{To cite this version:}

F. Doumenc, V. Janeček, V. S. Nikolayev. Thin wedge evaporation/condensation controlled by the vapor dynamics in the atmosphere. European Physical Journal E: Soft matter and biological physics, 2018, 41 (12), pp.147. 10.1140/epje/i2018-11758-8 . hal-01973904

\section{HAL Id: hal-01973904 \\ https://hal.sorbonne-universite.fr/hal-01973904}

Submitted on 8 Jan 2019

HAL is a multi-disciplinary open access archive for the deposit and dissemination of scientific research documents, whether they are published or not. The documents may come from teaching and research institutions in France or abroad, or from public or private research centers.
L'archive ouverte pluridisciplinaire HAL, est destinée au dépôt et à la diffusion de documents scientifiques de niveau recherche, publiés ou non, émanant des établissements d'enseignement et de recherche français ou étrangers, des laboratoires publics ou privés. 


\title{
Thin wedge evaporation/condensation controlled by the vapor dynamics in the atmosphere
}

\author{
F. Doumenc ${ }^{1,2}$, V. Janeček ${ }^{1, a}$, and V. S. Nikolayev ${ }^{3}$ \\ ${ }^{1}$ Laboratoire FAST, Univ. Paris-Sud, CNRS, Université Paris-Saclay, F-91405, Orsay, France \\ ${ }^{2}$ Sorbonne Université, UFR 919, 4 place Jussieu, F-75252, Paris Cedex 05, France \\ ${ }^{3}$ Service de Physique de l'Etat Condensé, CEA, CNRS, Université Paris-Saclay, CEA Saclay, 91191 Gif-sur-Yvette Cedex, France
}

\begin{abstract}
Evaporation or condensation in the vicinity of the immobile (pinned) contact line in an atmosphere of some inert (noncondensable) gas is considered here in a partial wetting configuration. Such a problem is relevant to many situations, in particular to a drop or a liquid film drying in open air. The thermal effects are not important and the mass exchange rate is controlled by the vapor dynamics in the gas. By following previous works, we account for the weak coupling between the diffusion in the gas and flow in the liquid through the Kelvin effect. Such a problem is nonlocal because of the diffusion in the gas. For generality, we consider a geometry of a liquid wedge posed on a flat and homogeneous substrate surrounded by a gas phase with a diffusion boundary layer of uniform thickness $\Lambda$. Similarly to the moving contact line problem, the phase change leads to the hydrodynamic contact line singularity. The asymptotic analysis of this problem is carried out for the liquid wedge of the length $L \gg \Lambda$. Three asymptotic regions are identified: the microscopic one (in which the singularity is relaxed, in the present case with the Kelvin effect) and two intermediate regions. The Kelvin effect alone turns to be sufficient to relax the singularity. The scaling laws for the interface slope and mass evaporation/condensation flux in each region are discussed. It is found that the difference of the apparent contact angle (i.e. interface slope in the second intermediate region) and the equilibrium contact angle is inversely proportional to the square root of $\Lambda$ and square root of the microscopic length, whatever is the singularity relaxation mechanism.
\end{abstract}

\section{Introduction}

Evaporation and condensation phenomena are met widely both in everyday life and in various industrial processes. From physicist's point of view, there are two practically important limiting cases of the mass exchange,depending on which mechanism limits the phase change rate. The first is the phase change controlled by the heat transfer in the liquid phase, where the liquid is in contact with its pure vapor [1-3]. During evaporation, the liquid-covered solid substrate is overheated with respect to the saturation temperature and the heat coming from the substrate is consumed as the latent heat at the liquid-gas interface. During condensation, the heat flow is opposite. This case of strong mass exchange is met in various heat exchangers (boiling heat exchangers, heat pipes, etc.). The second limiting case is that of a generally weaker mass exchange with an atmosphere of some inert (noncondensable) gas $[4,5]$. A common example is the drop drying in the open air. The thermal effects are much less important

a Present address: Department TSIE: Microgravity Payloads, Airbus Defence \& Space, 88039 Friedrichshafen, Germany because the slowest (thus limiting) process is the vapor dynamics in the diffusion layer formed in the gas phase. Many researchers worked in this important field. A significant progress has been achieved by Deegan et al [4] who have obtained the stationary diffusion problem solution in the half-space above a spherical-cap-shaped sessile liquid droplet posed on a solid substrate with the known macroscopic (i.e. apparent) contact angle. Many theories based on this solution have been developed afterwards, one can cite [6-11] among many others. The success of the Deegan et al solution is due to the integrability of the resulting local evaporation flux so that the total evaporation rate can be calculated and compared to the experiment. However, an incoherency appears in the liquid phase hydrodynamic problem. As discussed in sec. 3 below, such an evaporation flux would lead to the hydrodynamic stress that diverges at the contact line in a non-integrable way, similarly to what happens in the isothermal contact line motion problem [12]. Such a divergence is nonphysical. On the one hand, the total viscous dissipation becomes infinite, which implies the infinite energy. On the other hand, in a problem with a free interface it would lead to the infinite interface slope. One knows that the singular- 
ity is possible to relax by introducing some microscopicscale phenomena like precursor film [10], hydrodynamic slip [13] or Kelvin effect $[14,15]$. The macroscopic scale solution is obtained by matching the solutions in microscopic and macroscopic regions [16]. The introduction of an intermediate length scale turns out to be necessary. A remarkable feature of such a problem is that the form of the macroscopic solution (called Cox-Voinov formula) is independent of the microscopic mechanism that conditions only two constants, the Voinov length and angle.

In the case of pure vapor and latent-heat-controlled evaporation, the microscopic mechanisms have been extensively studied by many authors starting from Potash and Wayner [1]. These studies showed that the apparent contact angle grows with the evaporation intensity (see, e.g. [2, 3, 17-20] among many others). The theoretical results have been confirmed experimentally [21, 22]. The independence of the results of the microscopic model has been shown numerically by comparing the apparent contact angle for different microscopic mechanisms but the same fluid parameters [23]. We do not discuss this evaporation regime below. Instead, we address the diffusioncontrolled evaporation regime that was less studied.

It has been shown experimentally [24] that the increase of apparent contact angle with evaporation rate exists in this case too. In earlier approaches [6-9], the evaporation flux distribution along the interface was imposed independently of the interface shape. The full coupling of the problems in the vapor and liquid phases through the Kelvin effect is studied in more recent works $[10,11,25]$. The problem is more complicated in this case because of the non-locality of evaporation flux: the evaporation rate at one point of the interface depends on the vapor concentration distribution over the whole liquid-vapor interface. However the Kelvin effect is important as it provides zero evaporation flux at the immobile contact line [20] in the case of partial wetting (the case considered here), which conforms to the complete wetting case.

In the continuity of the above cited works, our purpose is to study the effect of the apparent contact angle increase under evaporation. This objective was targeted by several groups. Berteloot et al [8] proposed an approximate solution for an infinite liquid wedge on a solid substrate using the expression for the evaporation flux given by Deegan et al [4] thus decoupling the diffusive and hydrodynamic parts of the problem. The singularity is avoided by assuming a finite liquid height at a microscopic cut-off distance, imposed a priori. Eggers and Pismen [10] have considered the vapor phase and liquid phase problems coupled via the concentrational Kelvin effect for an axisymmetric drop geometry. They considered the complete wetting conditions and the precursor film.

By following our preceding work [26] devoted to the moving contact line, we consider a partial wetting configuration with a 2D geometry suitable, e.g., for drying of the liquid films deposited on the Wilhelmy plate [27]. We consider a diffusive layer of thickness $\Lambda$ above the thin liquid wedge-like film. In a real process the value of $\Lambda$ would depend on the characteristic of the flow (diffusion, natural

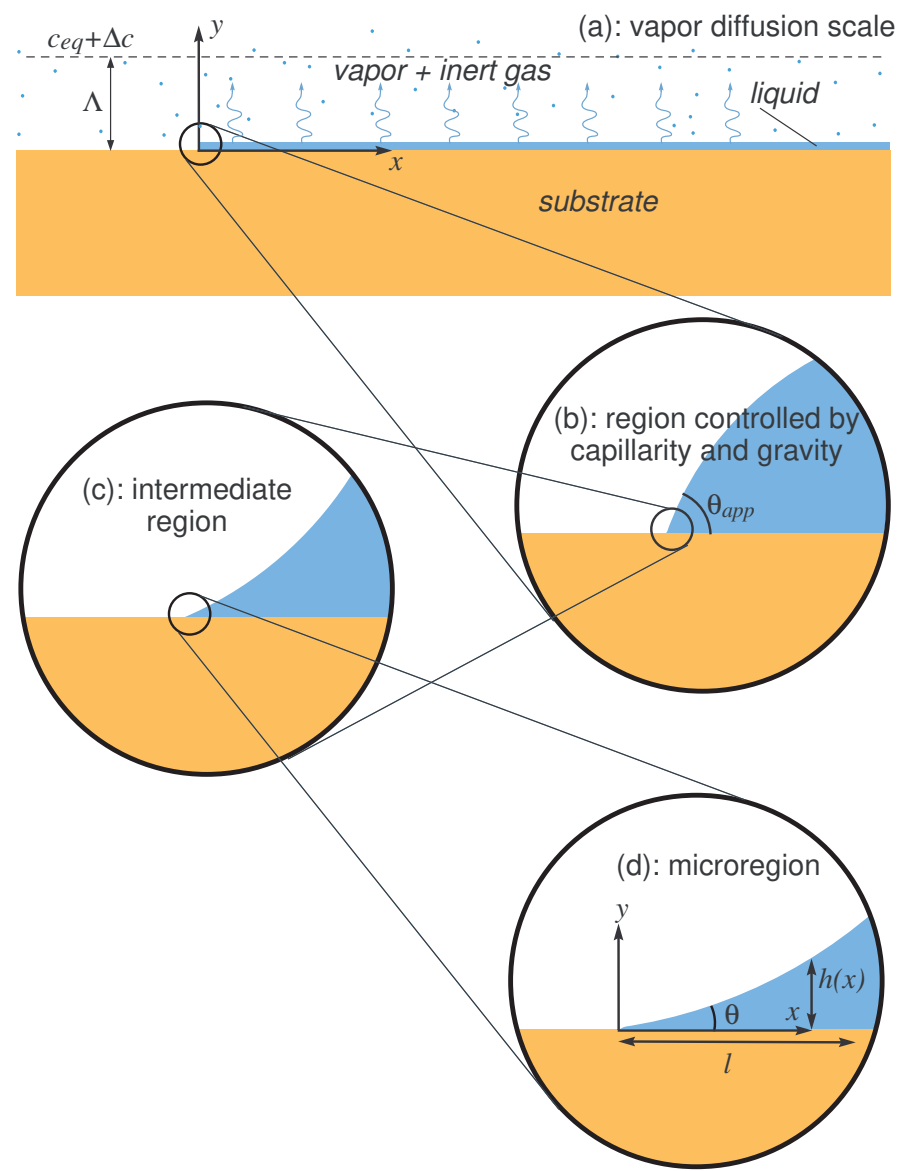

Fig. 1: Hierarchy of scales considered in the article and geometries for the vapor diffusion (a) and hydrodynamic (b-d) problems. Note that the radius of curvature in (b) is assumed to be much larger than the diffusive boundary layer width $\Lambda$ shown in (a).

convection or forced convection). We consider also the kinetic interfacial resistance effect that appears to be important in many cases. Using the Kelvin effect as microscopicscale phenomenon to relax the contact line singularity, we develop a complete description of the micro and intermediate regions (cf. fig. 1 and sec. 2). The influence of $\Lambda$ is analyzed, and a law for the apparent contact angle is deduced. Although the regularisation by the Kelvin effect may not apply to common fluids, (the micro-region size being too small for a continuum hydrodynamic approach [26]), the scalings characterising the intermediate region are independent of the mechanism chosen to relax the singularity. We thus draw results of general significance for the scaling of the apparent contact angle, since they are valid for any microscopic-scale phenomenon.

\section{Problem statement}

The problem to be considered is a thin liquid wedgeshaped film posed on a flat and homogeneous substrate in a situation of partial wetting. The atmosphere of a 
F. Doumenc et al.: Thin wedge evaporation/condensation controlled by the vapor dynamics in the atmosphere

non-condensable inert gas surrounds the substrate and the condensation or evaporation mass exchange with it is controlled by the vapor transfer in the gas. The gas supersaturation with vapor causes condensation onto the film, and under-saturation causes its evaporation. The fluid is assumed to be isothermal, which may be justified when the substrate has a good thermal conductivity and is maintained at room temperature. Such a situation is common, e.g. in the case of slow drying of liquid sessile drops. The contact line is assumed pinned, with a source/sink of liquid at $x=\infty$ that compensates the evaporation/condensation mass flux (the problem of contact line motion under saturation conditions has been solved in our preceding work [26]). The model considers the vapor diffusion in the gas phase, as well as the kinetic interfacial resistance.

First, some assumptions about the characteristic scale hierarchy need to be made. One can identify a macroscopic length scale $L$ that concerns the liquid phase. It is related to the macroscopic-level interface curvature, e.g. the interface curvature radius. Another macroscopic length scale concerns the gas phase description. It is the diffusion boundary layer thickness $\Lambda$. It is millimetric (for natural convection) or smaller, for forced convection. For simplicity, in this work we consider the diffusion boundary layer of homogeneous thickness (band-like). The idea of our approach is to find a stationary solution for the vapor diffusion that can be representative of real microscopic situation. For this we assume that

$$
L \gg \Lambda \gg h
$$

where $h$ is the wedge thickness, see fig. 1a,b. It is evident that the inequality (1) is violated far enough from the wedge apex (contact line) for any wedge slope. However, if the slope is small, the region of validity of such a geometry is large.

At a distance $\Lambda$ from the substrate, a deviation from the concentration corresponding to saturation is imposed. The main rationale of such a geometry is to decouple the scaling related to the macroscopic scale $L$ from dependencies on other length scales that exist in this problem so that our approach is applicable for different macroscopic geometries (drop, film, bubble, etc.), provided that inequality (1) is verified. Consequently, the liquid domain length $L$ must be much larger than the boundary layer thickness $\Lambda$. This excludes, for instance, the case of a sessile drop evaporating in quiescent air (no convection) [7], because in that case $\Lambda \sim L$.

A hydrodynamic singularity that exists in our problem is solved at the microscopic scale that we call $\ell$ which is typically nanometric (fig. 1d). Because of the existence of several characteristic lengths, intermediate regions (fig. 1c) are needed to match micro and macro regions. We will see later that, generally, two intermediate regions need to be introduced.

\subsection{Liquid phase equations}

Stokes equations (negligible inertia) are solved in lubrication approximation (small interface slopes) and neglect- ing the gravity. With no-slip boundary condition at the substrate and stress-free boundary condition at the interface, the horizontal component of the liquid velocity for $0<y<h, h$ being the liquid height, reads

$$
v_{x}(y)=\frac{\sigma}{\mu}\left(h y-\frac{1}{2} y^{2}\right) \frac{d \kappa}{d x},
$$

where $\sigma$ is the surface tension and $\kappa=d^{2} h / d x^{2}$ is the local interface curvature, $\mu$ the liquid viscosity and the coordinate system is shown in fig. $1 d$.

The fluid volume flux in the liquid layer vertical crosssection, $q(x)$, is

$$
q(x)=\int_{0}^{h} v_{x}(y) d y=\frac{\sigma h^{3}}{3 \mu} \frac{d \kappa}{d x} .
$$

The governing equation in the liquid phase is obtained by writing the mass conservation

$$
\rho \frac{d q}{d x}=-j
$$

where $\rho$ is the liquid density and $j$ is the interfacial mass flux ( $j>0$ for evaporation). From eqs. (3-4) one deduces

$$
\frac{d}{d x}\left(\frac{\sigma h^{3}}{3 \mu} \frac{d \kappa}{d x}\right)=-\frac{j}{\rho} .
$$

The interfacial mass flux is assumed to be controlled by the vapor diffusion in the inert gas:

$$
j=-\left.D_{g} \frac{\partial c}{\partial y}\right|_{y=0},
$$

where $c(x, y)$ is the vapor concentration (expressed in $\mathrm{kg} / \mathrm{m}^{3}$ ) and $D_{g}$ is the vapor diffusion coefficient.

Boundary conditions for the liquid phase are the following:

- For $x=0$ (contact line):

$$
h=0, \frac{d h}{d x}=\theta,
$$

where $\theta$ is the equilibrium contact angle imposed by intermolecular interactions at microscale.

- For $x \rightarrow \infty$, the interface curvature vanishes,

$$
\kappa=0 \text {. }
$$

\subsection{Gas phase model}

In the gas phase, the equation for vapor diffusion reads

$$
\frac{\partial^{2} c}{\partial x^{2}}+\frac{\partial^{2} c}{\partial y^{2}}=0
$$

In the framework of the small wedge slope approximation, the liquid gas interface seen from the large scale of the gas atmosphere is assumed to coincide with the line $y=0, x \geq$ 
0 , as shown in fig. 1a. This assumption is valid for a liquid height much lower than the boundary layer thickness, i.e. for $x \ll \Lambda / \theta$. As $\theta$ is a small angle, the model is valid over a distance to the contact line much larger than $\Lambda$.

The boundary conditions in partial wetting configuration are:

- For $y=\Lambda$ :

$$
c(x, \Lambda)=c_{e q}+\Delta c,
$$

where $c_{e q}$ is the vapor concentration at thermodynamic equilibrium for a flat liquid-gas interface, $\Delta c$ is the deviation from the equilibrium vapor concentration $(\Delta c<$ 0 corresponds to evaporation, while $\Delta c>0$ results in condensation).

- For $y=0$ and $x<0$ :

$$
\frac{\partial c}{\partial y}=0
$$

- The vapor concentration $c_{i}=c(x, y=0)$ at the liquidgas interface can deviate from $c_{e q}$ because of two reasons. First, the local equilibrium value $c_{i, e q}$ can be different from $c_{e q}$ because of the interface curvature (Kelvin effect). It will be considered later on. Second, $c_{i}$ can differ from $c_{i, e q}$ because of the interfacial resistance $R_{i}$, so that the following condition is valid for $x \geq 0$ :

$$
c_{i}=c_{i, e q}-j R_{i} .
$$

The interfacial resistance appears because of the molecula kinetic effects and is given by the Hertz-Knudsen relation $[28,29]$,

$$
R_{i}=\frac{2-f}{2 f} \sqrt{\frac{2 \pi M}{R_{g} T}},
$$

where $f$ is the accommodation factor close to unity [28], $R_{g}$ the ideal gas constant, $T$ the temperature, and $M$ the molar mass.

\section{Hydrodynamic contact line singularity related to the mass exchange}

To analyze the contact line singularity due to the internal flow, we drop temporarily the microscopic (Kelvin) effect so that $c_{i, e q}=c_{e q}$ in eq. (12).

First, consider the simplest case dominated by the kinetic resistance, where the concentration difference caused by the diffusion in the gas phase is much smaller than the kinetic resistance effect. The concentration gradient in the gas is then negligibly small and the concentration $c_{i}$ at the liquid interface is equal to the concentration at the upper edge of the diffusion boundary layer $y=\Lambda: c_{i}=c_{e q}+\Delta c$; $c_{i}$ is thus constant. As a consequence, the evaporation flux $j=\Delta c / R_{i}$ is constant $[c f$. eq. (12)].

From eqs. (2-4), the tangential stress at the substrate reads

$$
\left.\mu \frac{\partial v_{x}}{\partial y}\right|_{y=0}=-\frac{3 \mu j x}{h^{2} \rho} .
$$

Using $h \sim \theta x$, it follows that the tangential stress at the substrate scales as $x^{-1}$ for the configuration dominated by the kinetic resistance. It diverges at the contact line. The integral, i.e. the force acting on the substrate, diverges as $\ln (x)$, which is nonphysical. In reality, this quantity should be finite. In addition, the curvature diverges as $x^{-1}$, which means that the resulting slope (=apparent contact angle, the integral of curvature) is infinite, which is also nonphysical.

A similar analysis can be performed for the integral viscous dissipation

$$
\mu \int_{0}^{h}\left(\frac{\partial v_{x}}{\partial y}\right)^{2} d y=\frac{3 \mu(j x)^{2}}{h^{3} \rho^{2}},
$$

which diverges as $x^{-1}$ like the tangential stress. This is also nonphysical because it leads to the infinite energy losses.

These non-integrable divergences both for the tangential stress and viscous dissipation are similar to those of Huh and Scriven [12] in their seminal article about the moving liquid wedge with no evaporation.

Consider now the opposite case, i.e. negligible kinetic resistance and evaporation limited by diffusion in the gas phase. The solution for such a 2D problem [equations (912) with $c_{i, e q}=c_{e q}$ ] can be obtained by analogy with an electrostatic problem, cf. Appendix A. From eq. (A.12) [cf. its asymptotics (42) below], it follows that the evaporaation flux $j(x)$ scales as $x^{-1 / 2}$ in the vicinity of the contact line, in agreement with the Deegan's 3D axi-symmetric solution [4] applied for the vanishing contact angle. Accordingly, the tangential stress at the substrate scales as $x^{-3 / 2}$ and the total force diverges as $x^{-1 / 2}$. For the viscous dissipation and its integral, the scalings are $x^{-2}$ and $x^{-1}$, respectively. The curvature is non-integrable either (scales like $x^{-3 / 2}$ ).

We thus observe non-physical singularities in these two limiting cases, similarly to classical moving contact line problem. Note that the interfacial kinetic resistance alone does not relax the singularity in the hydrodynamic problem. Saxton et al [30] showed that regularization can be achieved by considering both interfacial resistance and hydrodynamic slip. In the present study, similarly to the moving contact line problem considered in our preceding work [26], the Kelvin effect is used to relax the contact line singularity.

\section{Kelvin effect and dimensionless formulation}

The Kelvin effect consists in the dependence of the equilibrium interfacial vapor concentration $c_{i, e q}$ on the interface curvature $\kappa$. In the first approximation this dependence reads $[10]$

$$
c_{i, e q}-c_{e q}=-\frac{M c_{e q}}{\rho R_{g} T} \sigma \kappa .
$$

The microregion size $\ell$ ( $c f$. fig. 1) for the case of the singularity relaxation with the Kelvin effect can be obtained with a scaling analysis [26]. For the purely diffusion 
F. Doumenc et al.: Thin wedge evaporation/condensation controlled by the vapor dynamics in the atmosphere

regime (i.e. for $R_{i}=0$ ) the characteristic size of a region dominated by the Kelvin effect is

$$
\ell=\frac{1}{\rho} \sqrt{\frac{3 \mu M c_{e q} D_{g}}{\theta^{3} R_{g} T}} .
$$

In fact the diffusion regime corresponds to $R_{i} \ll \ell / D_{g}$. Note that in the opposite limit (that we call kinetic regime hereafter) where $\mathcal{R}=R_{i} D_{g} / \ell \gg 1$, the characteristic size is

$$
\ell_{R}=\ell / \mathcal{R}=\frac{3 \mu M c_{e q}}{\theta^{3} \rho^{2} R_{g} T R_{i}} .
$$

Estimations given in Table 1 of our preceding article [26] show that $\mathcal{R}$ varies strongly from one fluid to another and with the contact angle. For example, $\mathcal{R}=0.72$ for ethanol and $\theta=1^{\circ}$, while $\mathcal{R}=166$ for glycerol and $\theta=5^{\circ}$.

Throughout the rest of the paper, we choose $\ell$ to make the equations dimensionless. The dimensionless abscissa is then $\tilde{x}=x / \ell$ and, accordingly to the wedge geometry, the liquid height scales as $H=h /(\theta \ell)$. The dimensionless curvature is $\mathcal{K}=\ell \kappa / \theta$.

The characteristic scale $J$ for the mass flux $(\tilde{j}=j / J)$ is $J=\theta^{4} \sigma \rho /(3 \mu)$ and the dimensionless lubrication equation reads

$$
\frac{d}{d \tilde{x}}\left(H^{3} \frac{d \mathcal{K}}{d \tilde{x}}\right)=-\tilde{j}
$$

Concentration deviation is reduced as $\tilde{c}=\left(c-c_{e q}\right) / \mathcal{C}$, with

$$
\mathcal{C}=\frac{\rho \sigma \theta^{4} \ell}{3 \mu D_{g}},
$$

as obtained from eqs. (5-6). The dimensionless counterpart of eq.(16) reads

$$
\tilde{c}_{i, e q}=-\mathcal{K} .
$$

The two remaining dimensionless equations are the mass flux and interfacial concentration expressions:

$$
\begin{array}{r}
\tilde{j}=-\left.\frac{\partial \tilde{c}}{\partial \tilde{y}}\right|_{\tilde{y}=0}, \\
\tilde{c}(\tilde{y}=0) \equiv \tilde{c}_{i}=-\mathcal{K}-\tilde{j} \mathcal{R} .
\end{array}
$$

They serve as boundary conditions for the diffusion problem.

\section{Weak evaporation approximation}

The weak evaporation approximation $\Delta c \ll \mathcal{C}$ is considered here. The variables are expanded in a regular perturbation series in $\epsilon=\Delta c / \mathcal{C}$. At the zero order corresponding to thermodynamic equilibrium $(\epsilon=0), \mathcal{K}_{0}=0, H_{0}=\tilde{x}$, $\tilde{j}_{0}=0$ and $\tilde{c}_{0}=0$, thus

$$
\begin{aligned}
\mathcal{K} & =\epsilon \mathcal{K}_{1}+\cdots, \\
H & =\tilde{x}+\epsilon H_{1}+\cdots, \\
\tilde{j} & =\epsilon \tilde{j}_{1}+\cdots, \\
\tilde{c} & =\epsilon \tilde{c}_{1}+\cdots .
\end{aligned}
$$

Note that $\epsilon$ is negative during evaporation and positive during condensation while $\tilde{j}_{1}, H_{1}$ and $K_{1}$ are always negative.

With the scaling defined in sec. 4 , the first order lubrication equation reads

$$
\frac{d}{d \tilde{x}}\left(\tilde{x}^{3} \frac{d \mathcal{K}_{1}}{d \tilde{x}}\right)=-\tilde{j}_{1},
$$

with the following boundary conditions:

- For $\tilde{x}=0: H_{1}=0, H_{1}^{\prime}=0$,

- For $\tilde{x} \rightarrow \infty: \mathcal{K}_{1}=H_{1}^{\prime \prime}=0$.

The first order problem for the diffusion in the gas phase is

$$
\frac{\partial^{2} \tilde{c}_{1}}{\partial \tilde{x}^{2}}+\frac{\partial^{2} \tilde{c}_{1}}{\partial \tilde{y}^{2}}=0
$$

with the boundary conditions

- For $\tilde{y}=\tilde{\Lambda} \equiv \Lambda / \ell$ :

$$
\tilde{c}_{1}=1
$$

- For $\tilde{y}=0$ and $\tilde{x}<0$ :

$$
\frac{\partial \tilde{c}_{1}}{\partial \tilde{y}}=0
$$

- For $\tilde{y}=0$ and $\tilde{x} \geq 0$ :

$$
\tilde{j}_{1}=-\frac{\mathcal{K}_{1}+\tilde{c}_{i, 1}}{\mathcal{R}}=-\left.\frac{\partial \tilde{c}_{1}}{\partial \tilde{y}}\right|_{\tilde{y}=0} .
$$

In the following sections we focus on two limit cases corresponding to situations dominated either by kinetic interfacial resistance (sec. 6) or by diffusion of vapor in the gas (sec. 8). We are looking for a regular solution at $\tilde{y} \rightarrow 0$, with no divergencies.

\section{Kinetic regime $(\mathcal{R} \rightarrow \infty)$ : analytical solution}

We first consider the mass exchange controlled by kinetic resistance. In practice, this assumption applies for $\mathcal{R} \gg$ $\Lambda \gg 1$. These two inequalities ensure that $\tilde{c}_{i, 1} \simeq 1$.

The problem defined in sec. 5 can be solved analytically in the kinetic regime. Equation (28) reduces to

$$
\mathcal{K}_{1}=-1-\mathcal{R} \tilde{j}_{1}
$$

and eq. (24) reads

$$
\frac{d}{d \tilde{x}}\left(\tilde{x}^{3} \frac{d \tilde{j}_{1}}{d \tilde{x}}\right)=\frac{\tilde{j}_{1}}{\mathcal{R}} .
$$

With the boundary condition $\lim _{\tilde{x} \rightarrow \infty} \tilde{j}_{1}(\tilde{x})=-1 / \mathcal{R}$ [stemming from eq. (29)] and the condition of a regular solution at $\tilde{x} \rightarrow 0$, the solution of eq. (30) is

$$
\tilde{j}_{1}=-\frac{2}{\mathcal{R}^{2} \tilde{x}} K_{2}\left(\frac{2}{\sqrt{\mathcal{R} \tilde{x}}}\right),
$$




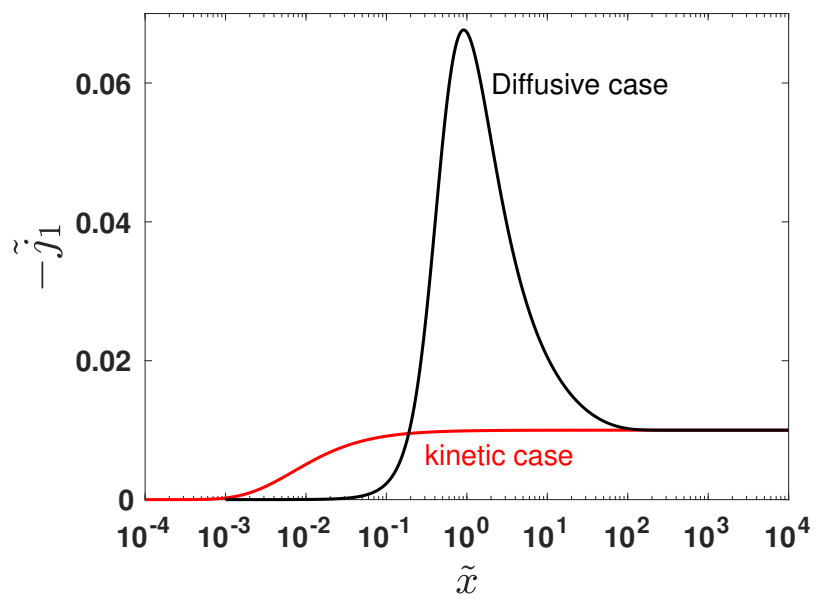

(a)

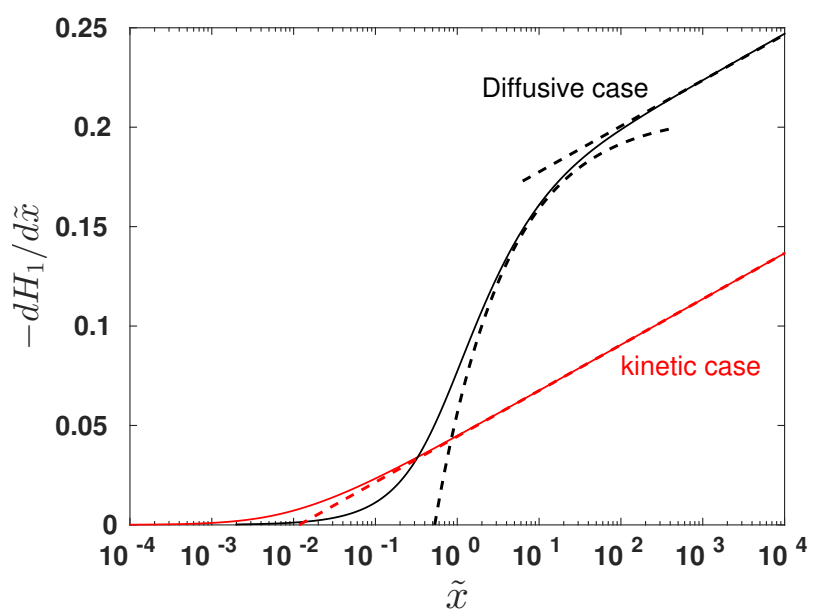

(b)

Fig. 2: (Color online) Comparison of kinetic [eqs. $(31,33)$ with $\mathcal{R}=100$, in red] and diffusive regimes $(\mathcal{R}=0$ and $\tilde{\Lambda}=100$ ); (a) flux curves and (b) slope curves. The dashed lines are the asymptotes coming from eq. (34) for the kinetic regime, eqs. (47) and (48) for the first and second intermediate subregions in the diffusive regime. In the latter case, panel (b) illustrates also the asymptotic matching of the first and second intermediate subregions.

where $K_{n}$ are the modified Bessel functions of the second kind.

The curvature $\mathcal{K}_{1}$ is then obtained from relation (29),

$$
\mathcal{K}_{1}=\frac{2}{\mathcal{R} \tilde{x}} K_{2}\left(\frac{2}{\sqrt{\mathcal{R} \tilde{x}}}\right)-1
$$

and the slope $d H_{1} / d \tilde{x}$ by integration of $\mathcal{K}_{1}$,

$$
\frac{d H_{1}}{d \tilde{x}}=2 \sqrt{\frac{\tilde{x}}{\mathcal{R}}} K_{1}\left(\frac{2}{\sqrt{\mathcal{R} \tilde{x}}}\right)-\tilde{x}
$$

The asymptotic behavior of the slope at infinite $\tilde{x}$ (i.e. in the intermediate region) is obtained from a series expan- sion of eq. (33)

$$
\frac{d H_{1}}{d \tilde{x}} \simeq-\frac{1}{\mathcal{R}} \ln \left(\frac{\tilde{x}}{\xi}\right),
$$

where $\xi=e^{2 \gamma-1} / \mathcal{R}$ and $\gamma \simeq 0.577216$ is the Euler Mascheroni constant.

An illustration of the kinetic regime is given for $\mathcal{R}=$ 100 in figs. 2 both for the flux and slope. Kelvin effect regularizes the solution. The flux does not diverge any more but drops over the dimensionless distance $\sim \xi$ from the contact line (i.e. dimensional distance $\sim \ell_{R}$ as expected for the kinetic regime). We obtain an expression similar to that of Cox-Voinov for the moving contact line [26]; the spatial variation of curvature is also similar. The characteristic length of the microscopic region is $\ell_{R} e^{2 \gamma-1}$. One notes that:

- this length is exactly the same as the Voinov length obtained for the moving contact line problem in the $\mathcal{R} \gg 1$ limit $[26]$;

- The dependence (34) is invariant of the microscale relaxation mechanism; only the expression for $\xi$ depends on it.

\section{Numerical solution for arbitrary $\mathcal{R}$}

For a finite $\mathcal{R}$, there is no analytical solution and the problem is solved numerically. Assuming that $\tilde{x}^{3} d \mathcal{K}_{1} / d \tilde{x} \rightarrow 0$ at $\tilde{x} \rightarrow 0$, integration of eq. (24) gives

$$
\tilde{x}^{3} \frac{d \mathcal{K}_{1}}{d \tilde{x}}=-\int_{0}^{\tilde{x}} \tilde{j}_{1} d \tilde{x} .
$$

The interfacial concentration $\tilde{c}_{i, 1}$ can be expressed as a function of the mass flux (see Appendix B):

$$
\tilde{c}_{i, 1}(\tilde{x})=1-\int_{0}^{\infty} G_{0}\left(\tilde{x}, \tilde{x}^{\prime}\right) \tilde{j}_{1}\left(\tilde{x}^{\prime}\right) d \tilde{x}^{\prime},
$$

with $G_{0}\left(\tilde{x}, \tilde{x^{\prime}}\right)$ given by eq. (B.14). Equation (28) thus reads

$$
\mathcal{K}_{1}=-1+\int_{0}^{\infty} G_{0}\left(\tilde{x}, \tilde{x}^{\prime}\right) \tilde{j}_{1}\left(\tilde{x}^{\prime}\right) d \tilde{x}^{\prime}-\mathcal{R} \tilde{j}_{1} .
$$

By its substitution into eq. (35), one gets the governing equation

$\tilde{x}^{3}\left(\int_{0}^{\infty} \frac{\partial G_{0}\left(\tilde{x}, \tilde{x}^{\prime}\right)}{\partial \tilde{x}} \tilde{j}_{1}\left(\tilde{x}^{\prime}\right) d \tilde{x}^{\prime}-\mathcal{R} \frac{d \tilde{j}_{1}}{d \tilde{x}}\right)=-\int_{0}^{\tilde{x}} \tilde{j}_{1}\left(\tilde{x}^{\prime}\right) d \tilde{x}^{\prime}$

that can be solved only numerically. Details of the numerical method are given in Appendix C. Once $\tilde{j}_{1}$ is known, concentration $\tilde{c}_{i, 1}$ and curvature $\mathcal{K}_{1}$ are obtained through equations (36) and (37), see Appendix $\mathrm{D} ; d H_{1} / d \tilde{x}$ and $H_{1}$ are computed by successive integrations of $\mathcal{K}_{1}$.

This numerical approach is used in the following section to study the situation dominated by diffusion of vapor in the gas. 


\section{Diffusion limited regime $(\mathcal{R}=0)$}

We analyze in this section the influence of $\tilde{\Lambda}$ on the contact line behavior, restricting the simulations to the purely diffusive case that corresponds to a situation such that $\mathcal{R} \ll 1 \ll \tilde{\Lambda}$.

Because of the presence of the lengthscale $\Lambda \gg \ell$, one identifies three asymptotic regions instead of two: the microregion dominated by the Kelvin effect and two intermediate regions where this effect is negligible. The first intermediate region ranges from 1 to $\tilde{\Lambda}$ in the dimensionless units, while the second goes from $\tilde{\Lambda}$ to infinity $(\tilde{L}$ in reality).

To describe both intermediate regions, the problem defined in sec. 5 is solved neglecting the Kelvin effect, which means $\tilde{c}_{i, e q}=0$. As mentioned in sec. 3 , the solution (A.12) can be obtained by analogy with an electrostatic problem, $c f$. Appendix A. The asymptotic expressions of this solution are obtained for both intermediate regions, assuming that the boundary layer thickness $\tilde{\Lambda}$ is much larger than the microregion size. The development obtained for $\tilde{x} \rightarrow 0$ within the $\tilde{c}_{e q, 1}=0$ assumption describes the first intermediate region $\tilde{x} \ll \tilde{\Lambda}$, while the limit $\tilde{x} \rightarrow \infty$ describes the second intermediate region such that $\tilde{\Lambda} \ll \tilde{x}$. It is however impossible to solve the microregion analytically so that numerical solution for all three regions will be found. Some scaling proper to the microregion is discussed.

\subsection{Asymptotic solutions for curvature in two intermediate regions}

We begin with the second intermediate region $\tilde{x} \gg \tilde{\Lambda}$, where the mass flux can be approximated by the expression stemming from eq. (A.12),

$$
\tilde{j}_{1}(\tilde{x}) \simeq-\frac{1}{\tilde{\Lambda}} .
$$

By using this expression in eq. (24), one obtains the curvature

$$
\mathcal{K}_{1}=-\frac{1}{\tilde{\Lambda} \tilde{x}}+\frac{\alpha_{1}}{\tilde{x}^{2}}+\alpha_{2}
$$

with $\alpha_{1}$ and $\alpha_{2}$ two integration constants. Vanishing curvature at infinity imposes $\alpha_{2}=0$. Considering that eq. (40) is an asymptotic solution for $\tilde{x} \rightarrow \infty$, we get

$$
\mathcal{K}_{1}(\tilde{x}) \simeq-\frac{1}{\tilde{\Lambda} \tilde{x}}
$$

at a leading order.

We turn now to the first intermediate region $\tilde{x} \ll \tilde{\Lambda}$, where the mass flux can be also obtained from eq. (A.12),

$$
\tilde{j}_{1}(\tilde{x}) \simeq-\frac{1}{\sqrt{\pi \tilde{\Lambda} \tilde{x}}} .
$$

This scaling was obtained previously for the thin 3D axisymmetric drop and infinite boundary layer [4]. However the present solution is different because the geometry is $2 \mathrm{D}$ and the liquid film is semi-infinite. For these reasons, the asymptotic solution for $\Lambda \rightarrow \infty$ is always $\Lambda$-dependent (see eq. (A.12) and the asymptotics $(39,42)$, where $j_{1} \rightarrow 0$ when $\Lambda \rightarrow \infty)$. Instead of $\Lambda$, the solution in ref. [4] contains the drop diameter.

The flux diverges at the contact line, as expected: it cannot describe the microregion dominated by the Kelvin effect. One gets the curvature after two successive integrations of eq. (24) where the expression (42) is used for $\tilde{j}_{1}$ :

$$
\mathcal{K}_{1}(\tilde{x}) \simeq-\frac{4}{3} \frac{1}{\sqrt{\pi \tilde{\Lambda} \tilde{x}^{3}}}+\frac{\alpha_{1}}{\tilde{x}^{2}}+\alpha_{2},
$$

containing the integration constants $\alpha_{1}$ and $\alpha_{2}$. Before going further, we need to determine which term in the rhs of eq. (43) is the leading order of the asymptotic development in the subregion $\tilde{x} \ll \tilde{\Lambda}$. The leading term order of magnitude estimated at the cross-over scale,

$$
M \equiv \max \left(\frac{1}{\tilde{\Lambda}^{2}}, \frac{\left|\alpha_{1}\right|}{\tilde{\Lambda}^{2}},\left|\alpha_{2}\right|\right),
$$

should match the order of magnitude of the solution (41), also estimated at the cross-over scale, i.e.

$$
M \sim \frac{1}{\tilde{\Lambda}^{2}} \quad \text { for } \quad \tilde{x} \sim \tilde{\Lambda} .
$$

First, we show that $\alpha_{2}$ can be neglected in eq. (43). The matching condition (45) cannot be satisfied if $\left|\alpha_{2}\right| \gg$ $1 / \tilde{\Lambda}^{2}$, since the definition (44) implies either $M \sim\left|\alpha_{2}\right| \gg$ $\frac{1}{\tilde{\Lambda}^{2}}$ or $M \gg\left|\alpha_{2}\right|$. Indeed, the first case is contradictory with eq. (45), which means $\left|\alpha_{2}\right| \lesssim 1 / \tilde{\Lambda}^{2}$. An immediate consequence is $\alpha_{2} \ll 1 / \sqrt{\tilde{\Lambda} \tilde{x}^{3}}$ for $\tilde{x} \ll \tilde{\Lambda}$ (because, for $\left.\tilde{x} \ll \tilde{\Lambda}, 1 / \tilde{\Lambda}^{2} \ll 1 / \sqrt{\tilde{\Lambda} \tilde{x}^{3}}\right)$. In other words, the first term in the r.h.s. of eq. (43) prevails asymptotically over the last which thus vanishes from the asymptotic solution.

Consider now the following asymptotic relation between the remaining two terms in eq. (43): $1 / \sqrt{\tilde{\Lambda} \tilde{x}^{3}} \ll$ $\left|\alpha_{1}\right| / \tilde{x}^{2}$. In this case, eq. (43) leads to $\left|\mathcal{K}_{1}(\tilde{x})\right| \sim\left|\alpha_{1}\right| / \tilde{x}^{2}$, and the matching condition (45) imposes $\left|\alpha_{1}\right| \sim 1$. We get $\left|\mathcal{K}_{1}(\tilde{x})\right| \sim 1 / \tilde{x}^{2}$ at leading order. This alternative is non-physical since the curvature is independent of $\tilde{\Lambda}$ when $\tilde{\Lambda} \rightarrow \infty$ (see the remark above on this issue). The only alternative is thus $\left|\alpha_{1}\right| / \tilde{x}^{2} \ll 1 / \sqrt{\tilde{\Lambda} \tilde{x}^{3}}$, which implies the condition $\alpha_{1}=0$ (we consider the subregion $\tilde{x} \ll \tilde{\Lambda}$ ). The asymptotic solution for the curvature in the first intermediate region is thus

$$
\mathcal{K}_{1}(\tilde{x}) \simeq-\frac{4}{3} \frac{1}{\sqrt{\pi \tilde{\Lambda} \tilde{x}^{3}}}
$$

One can see that the curvature (i.e., pressure) divergence is non-integrable at $\tilde{x} \rightarrow 0$ as expected for an intermediate region. 


\subsection{Asymptotic solutions for slope in the intermediate regions}

Integrating eq. (46) leads to the expression for the slope in the first intermediate region $(\tilde{x} \ll \tilde{\Lambda})$,

$$
\frac{d H_{1}}{d \tilde{x}} \simeq \frac{8}{3} \frac{1}{\sqrt{\pi \tilde{\Lambda}}}\left(\frac{1}{\sqrt{\tilde{x}}}-\frac{1}{\sqrt{\tilde{\eta}}}\right)
$$

with $\tilde{\eta}$ an integration constant. It is worth noting that a similar expression has been obtained by Berteloot et al [8]. However, both the "microscopic cut-off" length $\tilde{\eta}$ and the prefactor imposed ad hoc in ref. [8] are obtained rigorously within the present approach (see the next section for the $\tilde{\eta}$ determination).

The slope in the second intermediate region $(\tilde{x} \gg \tilde{\Lambda})$ is obtained by integration of relation (41):

$$
\frac{d H_{1}}{d \tilde{x}} \simeq-\frac{1}{\tilde{\Lambda}}\left[\ln \left(\frac{\tilde{x}}{\tilde{\Lambda}}\right)+C\right]
$$

with $C=-\tilde{\Lambda} \frac{d H_{1}}{d \tilde{x}}(\tilde{x}=\tilde{\Lambda})$ an integration constant. When $\tilde{\Lambda} \gg \tilde{\eta}$, the slopes (47) and (48) should match at the crossover $\tilde{x} \sim \tilde{\Lambda}$. This results in $C=k \sqrt{\tilde{\Lambda} / \tilde{\eta}}$ with $k$ a positive constant of the order unity. Both $\tilde{\eta}$ and $C$ are determined by matching to the microregion model in the next section.

Figures 2 shows the full numerical solution together with both asymptotic solutions $(47,48)$ to illustrate their validity.

\subsection{Numerical determination of constants in the asymptotic expressions}

The microregion asymptotic solution cannot be obtained analytically. Instead, the constants $\tilde{\eta}$ and $C$ that enter Eqs. $(47,48)$ are determined for $\mathcal{R}=0$ by solving numerically eq. (38) valid in all (microscopic and both intermediate) regions. The procedure described in Appendix $\mathrm{C}$ is used to solve such a complicated equation.

The slope perturbation $d H_{1} / d \tilde{x}$ is zero at the contact line since the contact angle is imposed at $\tilde{x}=0$. In the microregion, $d H_{1} / d \tilde{x} \sim-\tilde{x} / \sqrt{\tilde{\Lambda}}$ [see fig. 3b]. The $\tilde{\Lambda}^{-1 / 2}$ scaling is important to match the asymptotics (47) coming from the first intermediate region. The departure from the linear behavior corresponds to the end of microregion. Its size is of the order 1 in the dimensionless units ( $\ell$ in the dimensional), as expected.

The first intermediate region is described by eq. (47) that involves a characteristic length $\tilde{\eta}$. It can be obtained numerically by plotting the quantity

$$
\Gamma=3 / 8(\pi \tilde{\Lambda})^{1 / 2} \frac{d H_{1}}{d \tilde{x}}-\tilde{x}^{-1 / 2}
$$

as a function of $\tilde{x}$. This quantity is derived from eq. (47) and, according to it, is expected to be constant in the first intermediate region. According to the numerical solution (fig. 3a), this quantity shows the $\tilde{x}$-independent region (where $\Gamma \simeq-1.37$ ) only for $\tilde{\Lambda} \gg 1$. This is exactly the criterion of existence of the first intermediate region, cf. sec. 8. From the relation $\Gamma=-\tilde{\eta}^{-1 / 2}$ that comes from Eqs. $(47,49)$, one gets $\tilde{\eta} \simeq 0.53$, which is of the order of 1 as expected.

To analyze the large scale behavior (in the second intermediate region) one needs to renormalize the slope data of fig. 3. It is done in fig. 4a. A logarithmic variation of $d H_{1} / d \tilde{x}$ is observed for $\tilde{x} / \tilde{\Lambda}>1$, as predicted by eq. (48). The integration constant $C$ is obtained by fitting eq. (48) to the numerical results [see dashed lines in fig. 4a] and taking the intersection of each fit with the dash-dotted line $\tilde{x}=\tilde{\Lambda}$. The parameter $C$ is given in fig. $4 \mathrm{~b}$ as a function of $\tilde{\Lambda}$. For $\tilde{\Lambda} \gg 1$, we get $C=\beta \sqrt{\tilde{\Lambda}}$ with $\beta \simeq 2$ and the law $C=k \sqrt{\tilde{\Lambda} / \tilde{\eta}}$ indeed holds, as expected ( $c f$. sec. 8.2). By using $\tilde{\eta} \simeq 0.53$ obtained above, we get $k \simeq 1.46$.

\subsection{Apparent contact angle}

It is interesting to analyze the behavior of the apparent contact angle $\theta_{\text {app }}$, i.e. that observed at the macroscopic length scale $L$. Strictly speaking, the slope depends on $x$ so that $\theta_{a p p}$ can only be determined after asymptotic matching to a particular macroscopic geometry (drop, bubble, etc.). Such a procedure has been already performed, e.g. for the capillary dewetting [31]. However, some useful information about the apparent contact angle can be obtained from the present analysis. It can be taken from the slope in the second intermediate region.

Indeed, in the case of the thin thermal boundary layer and long film, $x$ can be in the second intermediate region $\Lambda \ll x \ll L$, where, from eq. (48), the slope varies logarithmically:

$$
\frac{d h}{d x} \simeq \theta-\frac{3 \mu D_{g} \Delta c}{\rho \sigma \theta^{3} \Lambda}\left[\beta \sqrt{\frac{\Lambda}{\ell}}+\ln \left(\frac{x}{\Lambda}\right)\right] .
$$

This expression means that the actual apparent contact angle value will slightly depend on the curvature in the macroscopic region, similarly to the moving contact line problem [31]. Neglecting the weak logarithmic dependence in eq. (50) yields an approximate expression of the apparent contact angle,

$$
\theta_{a p p} \simeq \theta-\frac{3 \beta}{\sqrt{\ell}} \frac{\mu D_{g}}{\rho \sigma \theta^{3}} \frac{\Delta c}{\sqrt{\Lambda}} .
$$

On the other hand, eq. (51) can also be obtained by tending $\tilde{x} \rightarrow \infty$ in eq. (47), i.e. within the first intermediate region. In this case one gets instead of $3 \beta \simeq 6$ the coefficient $8 / \sqrt{\pi \tilde{\eta}} \simeq 6.2$. Two values are very close, which is not surprising because both intermediate regions are matched (in the asymptotic sense) for $\tilde{x} \rightarrow \infty$ for the first and $\tilde{x} \rightarrow \tilde{\Lambda}$ for the second. The agreement shows simply that the matching has been performed correctly.

Note that eq. (51) is the result of the intermediate regions where microscopic effects are negligible (see eqs. (47)-(48)). Therefore, the form of eq. (51) is independent of the microscopic singularity relaxation mechanism. The 


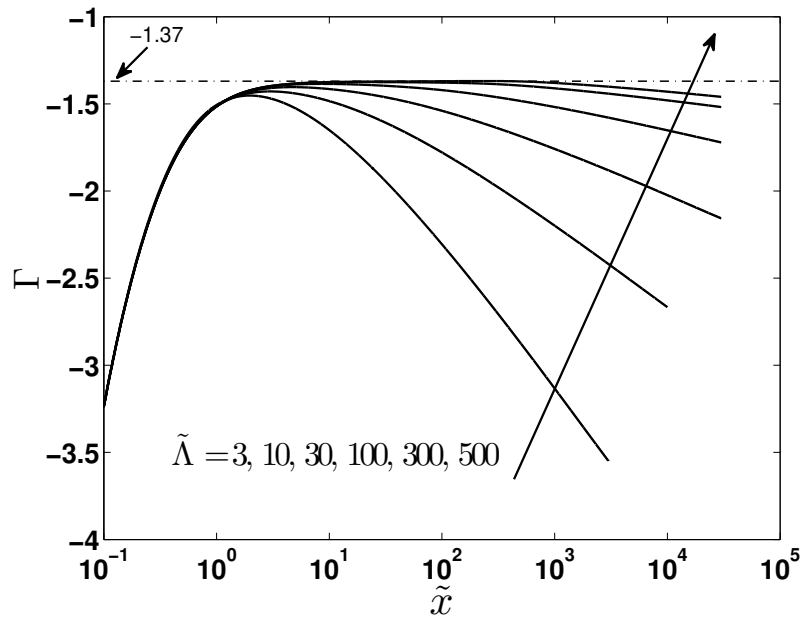

(a)

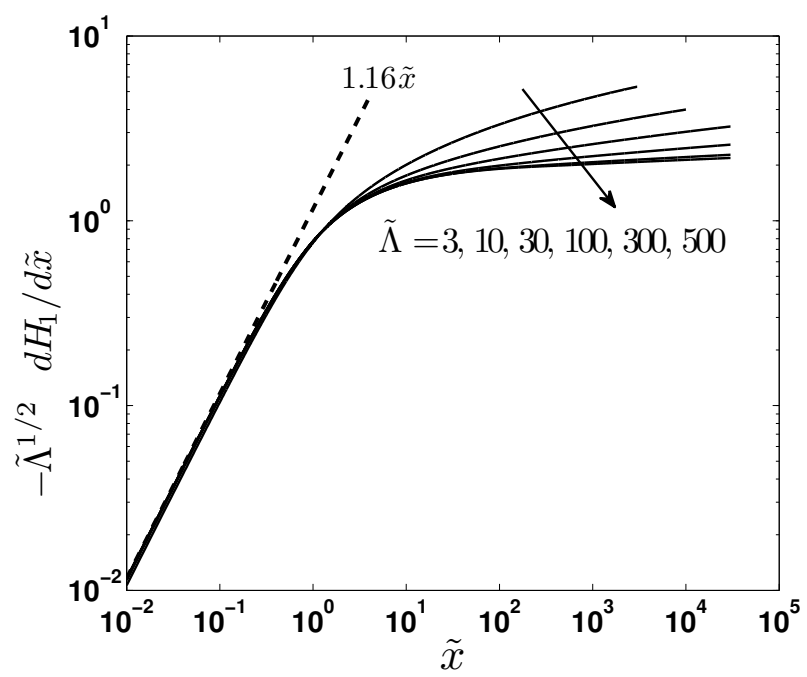

(b)

Fig. 3: Matching of microregion and first intermediate region. (a) Solid lines represent $\Gamma(\tilde{x})$ function obtained numerically; dash-dotted line is the asymptotic value $\Gamma \simeq$ -1.37 of the first intermediate region. (b) $-\tilde{\Lambda}^{1 / 2} d H_{1} / d \tilde{x}$; solid lines: numerical results; dashed line: asymptotic microregion behavior. The arrows show the directions of the $\tilde{\Lambda}$ increase.

microscopic details impact only the microregion length scale $\ell$. For instance, if the singularity was relaxed with the hydrodynamic slip length $\ell_{s}$, the characteristic length $3 \ell_{s} / e \theta$ (where $e=2.71 \ldots$ ) [32] would need to be used instead of $\ell$. If the singularity was relaxed with the precursor film, $\ell$ would scale on the Israelachvili length $(A / 6 \pi \sigma)^{1 / 2}$, where $A$ is the Hamaker constant.

Equation (51) is very close to the result of Berteloot et al [8] (one needs to drop the independent Cox-Voinov term in their eq. (13) to conform to the present problem statement; in addition, it should be noted that the boundary layer thickness $\Lambda$ is included in their parameter $J_{0}$ ). There are however differences between our approach and

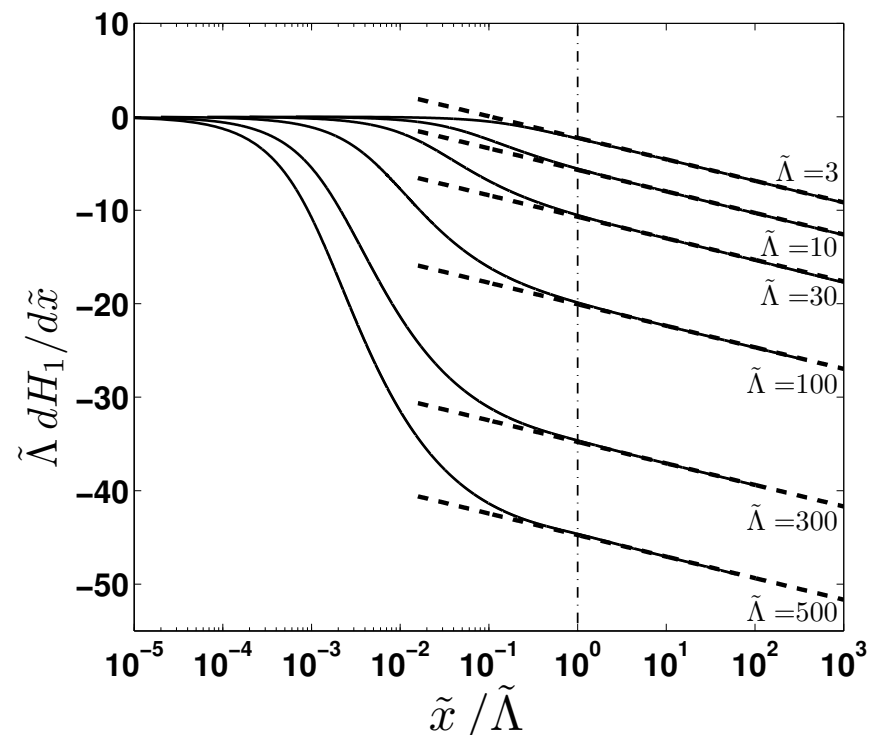

(a)

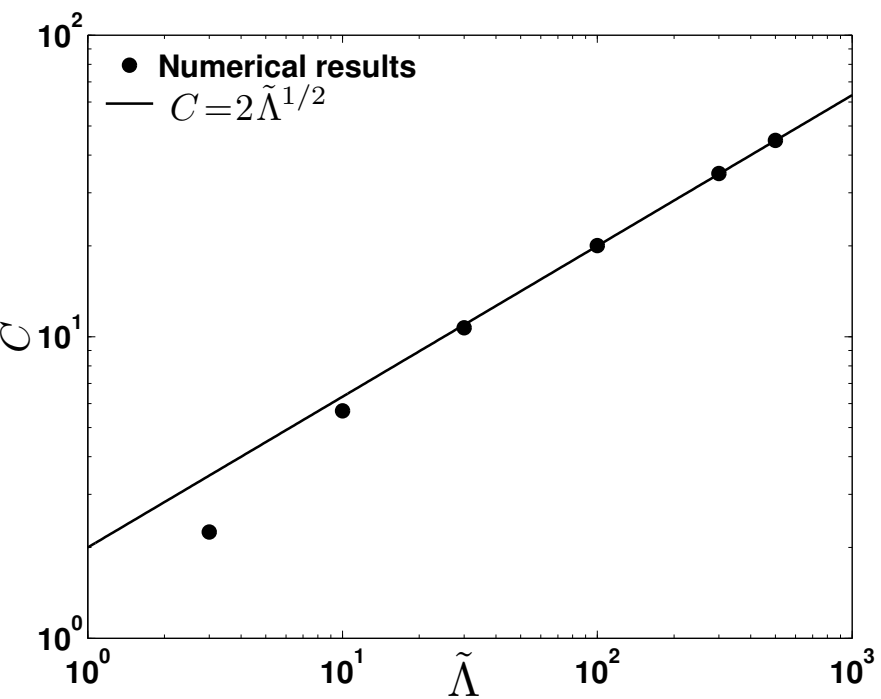

(b)

Fig. 4: Matching of the first and second intermediate regions. (a) $\tilde{\Lambda} d H_{1} / d \tilde{x}$ for different values of $\tilde{\Lambda}$; solid lines: numerical results; dashed lines: asymptotic equation (48); vertical dash-dotted line: $\tilde{x}=\tilde{\Lambda}$. Note that the microregion behavior cannot be seen on these curves (the scale is too large). (b) Integration constant $C$ as a function of $\tilde{\Lambda}$.

that of ref. [8]. Berteloot et al introduced the microscopic scale $\ell$ phenomenologically, and found the numerical prefactor $3 \beta$ of the second term equal to 8 . In our approach, the scales are matched rigorously, and a more precise value of 6 is obtained. In the work of Berteloot et al, only the first intermediate region $x \ll \Lambda$ is considered. The slope logarithmic variation in the second intermediate region is thus implicitely neglected.

The last but not least, within our approach, the local curvature and heat flux are obtained at all length scales 
including the microregion. Their behavior is discussed in the next section.

\subsection{Mass flux and curvature behavior}

Figures 5 displays the mass flux and curvature for $\tilde{\Lambda}$ varying over several orders of magnitude. First, one can see that both the mass flux and the curvature are regular at the contact line due to the account of the Kelvin effect.

The microregion corresponds to the domain $\tilde{x} \lesssim 1$, where the Kelvin effect is dominant and regularizes the singularity. Numerical results show that the flux $\tilde{j}_{1}$ varies in the microregion as $\tilde{x}^{2} \tilde{\Lambda}^{-1 / 2}$ [dash-dotted line in fig. 5a].

In the first intermediate region $1 \lesssim \tilde{x} \lesssim \tilde{\Lambda},-\tilde{\Lambda}^{1 / 2} \tilde{j}_{1}$ and $-\tilde{\Lambda}^{1 / 2} \mathcal{K}_{1}$ plotted as functions of $\tilde{x}$ gather on master curves [see figs. 5]. Similarly to the slope, one can see that the $\tilde{\Lambda}^{-1 / 2}$ scaling of other microregion variables matches that of their intermediate region counterparts [cf. equations (42) and (46)].

In the second intermediate region $\tilde{x} \gg \tilde{\Lambda}$, the flux goes to the constant value $-1 / \tilde{\Lambda}$ and the curvature behaves as $-1 /(\tilde{\Lambda} \tilde{x})$ (figs. 6$)$. As predicted by equations (39) and (41), the quantities $-\tilde{j}_{1} \tilde{\Lambda}$ and $-\mathcal{K}_{1} \tilde{\Lambda}^{2}$ plotted as functions of $\tilde{x} / \tilde{\Lambda}$ gather on master curves. A cross-over to this constant flux regime occurs at a distance of about $\tilde{\Lambda}$ to the contact line, as expected.

Finally, it is interesting to compare the behavior of the flux and slope for the purely kinetic and purely diffusive regimes, as depicted in figs. 2. The two parameters, $\mathcal{R}=100$ and $\tilde{\Lambda}=100$, have been chosen in order to obtain the same flux far from the contact line. First, the behavior of the evaporative flux is different. Diffusion in the gas induces a large peak close to the contact line, while a monotonic decrease is observed for the kinetic case. At large scale, both regimes show a logarithmic variation of the slope, but the diffusive regime exhibits also an additional (the first) intermediate region that does not exist for the kinetic regime.

\section{Conclusions}

Evaporation or condensation controlled by vapor diffusion in the atmosphere causes a hydrodynamic singularity in the vicinity of the edge of the liquid wedge, i.e. a nonintegrable divergence of the hydrodynamic stresses at the contact line, just like in the contact line motion case. The interfacial kinetic resistance does not lead to the singularity relaxation (this was first shown in ref. [30]). Another microscopic phenomenon thus needs to be introduced into the modeling of such a phenomenon to relax the singularity.

The singularity relaxation by Kelvin effect (a dependence of saturation vapor concentration on the interface curvature) is used to solve in a closed form a 2D problem of a thin liquid wedge in the atmosphere at given super-saturation $\Delta c$ in the presence of diffusive boundary

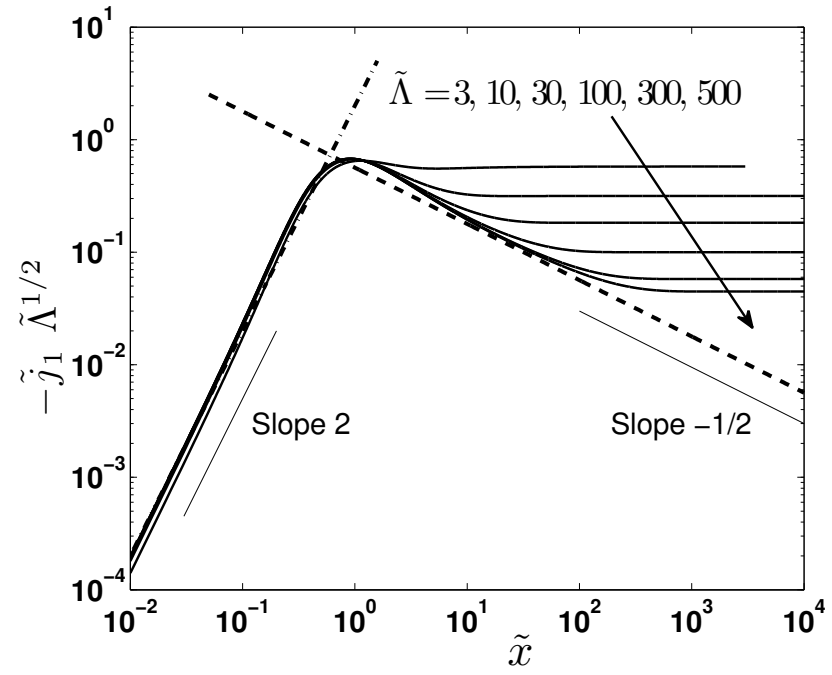

(a)

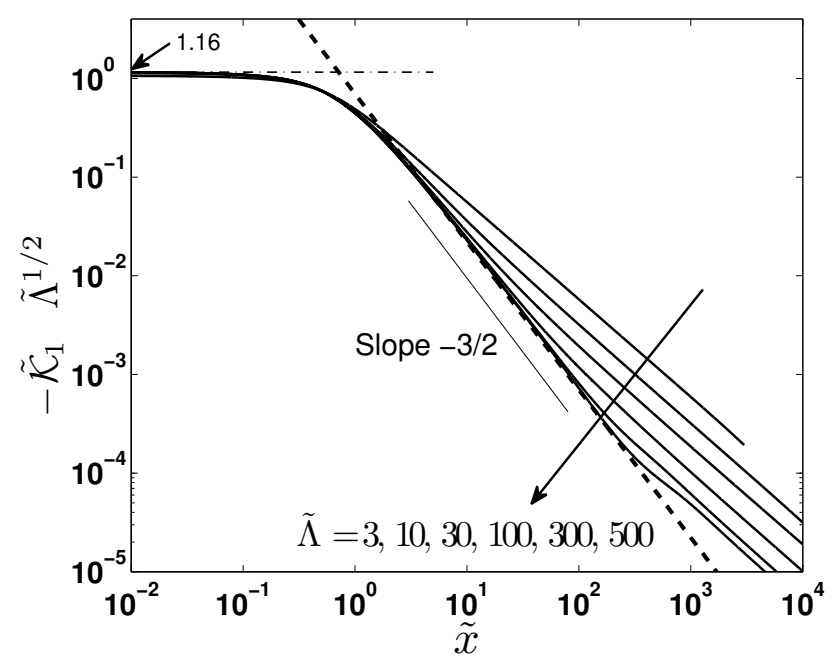

(b)

Fig. 5: Behavior of mass flux (a) and curvature (b) for different values of $\tilde{\Lambda}$. The arrows show the directions of the $\tilde{\Lambda}$ increase. The scaling is appropriate for microregion and first intermediate region. Dashed line: first intermediate region asymptotics (42) (a) and (46) (b). Dash-dotted lines: microregion behavior (fits to numerical curves).

layer of a finite thickness (the negative $\Delta c$ causes undersaturation of the atmosphere and thus liquid evaporation). We use the approximations of weak mass exchange, i.e. small $\Delta c$, and small wedge slope.

The numerical estimates for the size $\ell$ of the contact line vicinity, in which the Kelvin effect is important (the Kelvin length) can be found elsewhere [26]. They lead to the same conclusion as for the moving contact line case: for common fluids, the liquid height in the microregion is never much larger than $1 \mathrm{~nm}$, and much lower in some cases, which is not consistent with the continuum hydrodynamic approach. 


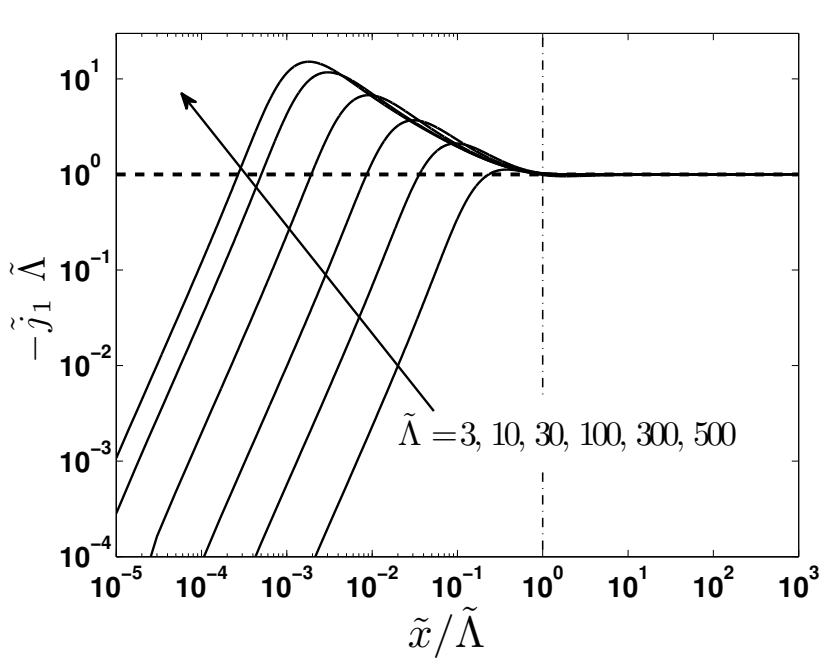

(a)

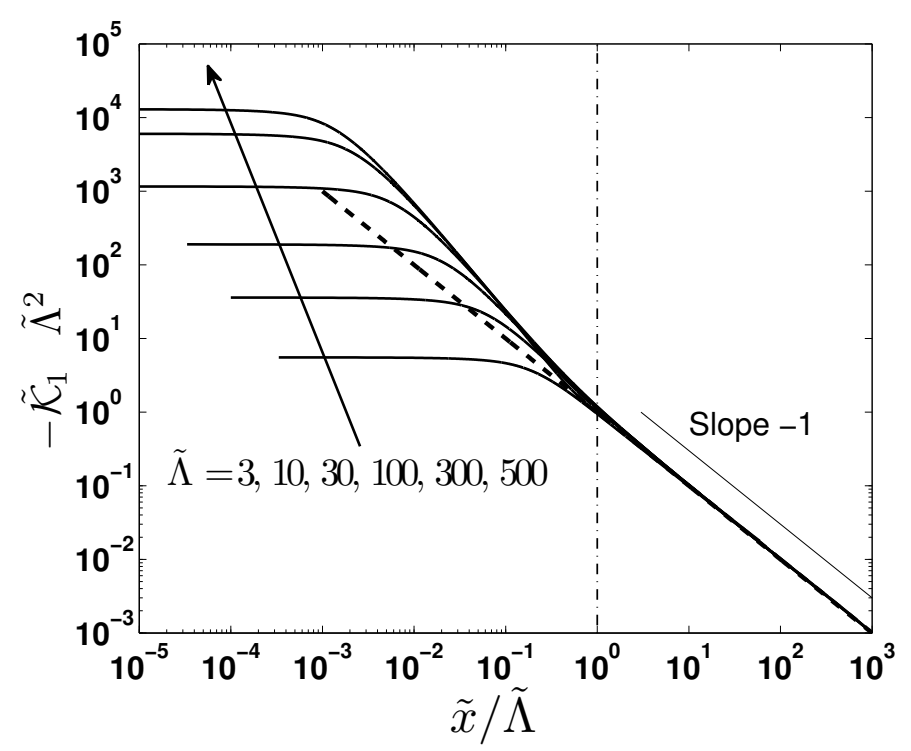

(b)

Fig. 6: Behavior of mass flux (a) and curvature (b) for different values of $\tilde{\Lambda}$. The arrows show directions of the $\tilde{\Lambda}$ increase. The scaling is appropriate for the second intermediate region. Dashed lines: asymptotics (39) (a) and (41) (b) for the second intermediate region. Vertical dashdotted lines correspond to $\tilde{x}=\tilde{\Lambda}$.

The macroscopic scaling laws are independent of the precise mechanism that ensures singularity relaxation. Due to scale separation, the intermediate region scalings remain valid if the singularity is relaxed with another microscale effect like hydrodynamic slip [3] or flow in a thin adsorbed liquid film adjacent to the wedge [10]; In the asymptotic expressions, only $\ell$ value depends on the microscopic details.

We consider two limiting cases: the kinetic case where the mass exchange rate is entirely controlled by the interfacial kinetic resistance $(1 \ll \tilde{\Lambda} \ll \mathcal{R})$, and the diffusive case where it is entirely controlled by the diffusion in the

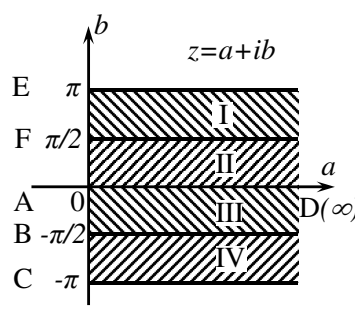

(a)

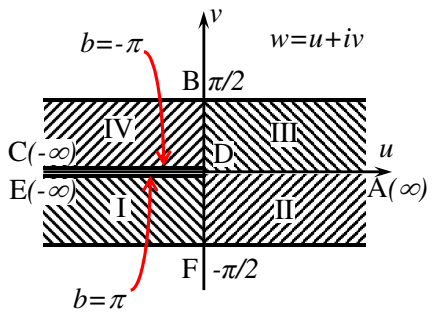

(b)
Fig. A.1: Conformal mapping: (a) $z$ domain, (b) $w$ domain. The corresponding points A,B,C,D,E,F and areas I,II,II,IV of the both domains are shown.

gas phase $(\mathcal{R} \ll 1 \ll \tilde{\Lambda})$. The kinetic case is solved analytically. The result is very similar to the Cox-Voinov solution for the moving contact line case, with exactly the same characteristic size $\ell$ of microscopic region.

In a general problem, there is another length scale: the thickness $\Lambda \gg \ell$ of the diffusion boundary layer. Because of this, two intermediate regions can be identified. For the first of them, $\ell \ll x \ll \Lambda$. For the second, $\Lambda \ll x \ll L$, where $L$ is the macroscopic length scale (drop or bubble size, capillary ridge width, etc.). The asymptotic scaling laws describing the mass flux, curvature and interface slope are derived for both intermediate regions. They agree with the numerical solutions of general problem.

It is found that the apparent contact angle is close to the value obtained by Berteloot et al. [8]. The prefactor in their equation should be however corrected. The precise value of the apparent contact angle depends weakly (logarithmically) on the macroscopic curvature (that of the drop or curved meniscus).

This work has been financially supported by the LabeX LaSIPS (ANR-10-LABX-0040-LaSIPS) managed by the French National Research Agency under the "Investissements d'avenir" program (ANR-11-IDEX-0003-02).

\section{Author contribution statement}

All the authors were involved in the problem statement, interpretation of results and manuscript review. FD and VJ performed the numerical simulations. VSN performed the analytical calculations.

\section{A Vapor diffusion from thin liquid wedge to the boundary layer}

One aims at solving the 2D Laplace equation for the vapor concentration $c$ produced by the evaporating zero thickness liquid wedge within the diffusive boundary layer, $c f$. fig. 1(a). The geometrical setup of the reduced problem is adapted to the already solved equivalent electrostatic 
problem where the potential is defined as $\varphi=\left(c-c_{e q}\right) / \Delta c$. The reduced coordinates are $(u, v)$ and the problem

$$
\begin{aligned}
\Delta \varphi(u, v) & =0, \\
\left.\varphi\right|_{v=\pi / 2} & =1, \\
\left.\frac{\partial \varphi}{\partial v}\right|_{v=0, u>0} & =0, \\
\left.\varphi\right|_{v=0, u<0} & =0
\end{aligned}
$$

is considered in the domain $-\infty<u<\infty, 0<v<\pi / 2$. Unlike the initial problem statement, the wedge situates to the left of the point $u=0$ so that the relations between the actual and reduced coordinates are $x=-2 u \Lambda / \pi$ and $y=2 v \Lambda / \pi$. A symmetric equipotential plate can be added at $v=-\pi / 2$ and the domain can be extended to $-\infty<$ $u<\infty,-\pi / 2<v<\pi / 2$, cf. figure A.1b. The boundary conditions

$$
\left.\varphi\right|_{v= \pm \pi / 2}=1
$$

replace the conditions (A.2, A.3) because the second of them is satisfied trivially (because of symmetry).

Such a domain may be conformally mapped to a halfband in the plane $(a, b)$ shown in figure A.1a, where $\varphi=1$ is imposed at the side $a=0,-\pi<b<\pi$, and $\varphi=0$ is at the sides $b= \pm \pi, 0<a<\infty$. The corresponding conformal mapping function [33] is

$$
z=\ln \operatorname{coth}\left(\frac{w}{2}\right) \equiv \ln \frac{e^{w}+1}{e^{w}-1},
$$

where $z=a+i b$ and $w=u+i v$. It is noteworthy that the inverse function is identical.

The solution

$$
\varphi=\frac{2}{\pi} \arctan \left[\frac{\cos (b / 2)}{\sinh (a / 2)}\right]
$$

of the Laplace problem for such half-band domain is known [34] (\$7.4, p. 47). From (A.6), one obtains

$$
\begin{aligned}
& a=\frac{1}{2} \ln \frac{\cosh u+\cos v}{\cosh u-\cos v}, \\
& b=\arctan (\sinh u,-\sin v),
\end{aligned}
$$

where the function $\arctan \left(x^{\prime}, y^{\prime}\right)$ gives the polar angle $-\pi \leq \alpha \leq \pi$ of a point $\left(x^{\prime}, y^{\prime}\right)$ (in programming languages it is often called atan2).

The substitution of equations (A.8,A.9) into (A.7) results in the complete solution of the problem (A.1-A.4).

We are interested in the evaporative flux (i.e. in the concentration gradient) at the liquid-gas interface $v \rightarrow$ $+0, u<0$ (line C-D in figure A.1b). Since both arguments in eq. (A.9) are negative in this case, eq. (A.9) reduces to

$$
b=-\pi-\arctan \left(\frac{\sin v}{\sinh u}\right),
$$

where $\arctan \left(x^{\prime}\right)$ is now defined for $-\pi / 2<x^{\prime}<\pi / 2$ as usually. One can see now that $b \rightarrow-\pi$ when $v \rightarrow 0$.
To find the derivative $\partial \varphi /\left.\partial v\right|_{v \rightarrow+0}$ at $u<0$, one writes

$$
\frac{\partial \varphi}{\partial v}=\frac{\partial \varphi}{\partial a} \frac{\partial a}{\partial v}+\frac{\partial \varphi}{\partial b} \frac{\partial b}{\partial v}
$$

and then tends $b \rightarrow-\pi$ and $v \rightarrow 0$. Equation (A.7) is used to calculate $\partial \varphi / \partial a$ and $\partial \varphi / \partial b$. The expression (A.10) needs to be used to calculate $\partial b / \partial v$. The result is

$$
\left.\frac{\partial \varphi}{\partial v}\right|_{l i q}=\frac{2}{\pi} \sqrt{\frac{1-\operatorname{coth}(u)}{2}} .
$$

In the notation of sec. 5 , the flux $\tilde{j}_{1}$ reads

$$
\tilde{j}_{1}(\tilde{x})=-\frac{1}{\tilde{\Lambda}} \sqrt{\frac{1}{2}\left[1+\operatorname{coth}\left(\frac{\pi \tilde{x}}{2 \tilde{\Lambda}}\right)\right]} .
$$

For $\tilde{x} \gg \tilde{\Lambda}$, eq. (A.12) results in eq. (39), which is simply a constant gradient solution for the boundary layer of the thickness $\tilde{\Lambda}$. For $\tilde{x} \ll \tilde{\Lambda}$, one obtains eq. (42).

\section{B Governing equation for the vapor concentration field}

The tilde denoting dimensionless quantities is omitted in this appendix for clarity.

The starting point for the derivation is Green's second identity

$$
\begin{aligned}
& \int_{\mathcal{D}}\left[\varsigma(\boldsymbol{r}) \Delta_{r} G\left(\boldsymbol{r}, \boldsymbol{r}^{\prime}\right)-G\left(\boldsymbol{r}, \boldsymbol{r}^{\prime}\right) \Delta \varsigma(\boldsymbol{r})\right] d \boldsymbol{r}= \\
& \oint_{\mathcal{L}}\left[\varsigma(\boldsymbol{r}) \nabla_{r} G\left(\boldsymbol{r}, \boldsymbol{r}^{\prime}\right)-G\left(\boldsymbol{r}, \boldsymbol{r}^{\prime}\right) \nabla \varsigma(\boldsymbol{r})\right] \cdot \boldsymbol{n} d l_{r},
\end{aligned}
$$

where $G$ is the Green function, $\varsigma=c_{1}-1$, and $\mathcal{L}$ is the boundary of the domain $\mathcal{D}$ with the outward unit normal $\boldsymbol{n}$. The boundary consists of the $x$ axis $\mathcal{L}_{x}$ and the line $\mathcal{L}_{\Lambda}$ defined by $y=\Lambda$ (the integrals over the infinitely distant vertical parts of the contour are zero, which can be easily checked with the Green function derived below). The differentiation in all differential operators is assumed hereafter to be performed over the components of the vector $\boldsymbol{r}$ rather than those of $\boldsymbol{r}^{\prime}$. The equation for $\varsigma$ is

$$
\Delta \varsigma=0
$$

with the boundary conditions

$$
\begin{aligned}
& \left.\frac{\partial \varsigma}{\partial y}\right|_{y=0, x<0}=0, \\
& \left.\frac{\partial \varsigma}{\partial y}\right|_{y=0, x>0}=-j_{1}(x), \\
& \left.\varsigma\right|_{y=\Lambda}=0 .
\end{aligned}
$$

The corresponding to this problem Green function $G=$ $G\left(\boldsymbol{r}, \boldsymbol{r}^{\prime}\right)$ satisfies the equation

$$
\Delta G\left(\boldsymbol{r}, \boldsymbol{r}^{\prime}\right)=\delta\left(\boldsymbol{r}-\boldsymbol{r}^{\prime}\right)
$$




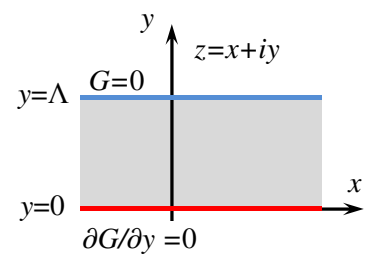

(a)

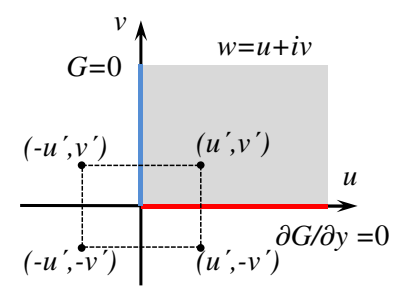

(b)
Fig. B.1: Domain for the Green function problem in the $z$ plane (a) and its conformal map, the $w$ plane (b).

Its general solution in $2 \mathrm{D}$ is

$$
G\left(\boldsymbol{r}, \boldsymbol{r}^{\prime}\right)=\frac{1}{2 \pi} \ln \left|\boldsymbol{r}-\boldsymbol{r}^{\prime}\right|+H\left(\boldsymbol{r}, \boldsymbol{r}^{\prime}\right),
$$

where $H$ satisfies the equation $\Delta H=0$ in $\mathcal{D}$ so it is nonsingular when $\boldsymbol{r}=\boldsymbol{r}^{\prime}$. It is determined from the boundary conditions

$$
\left.(\boldsymbol{n} \cdot \nabla) G\right|_{\boldsymbol{r} \in \mathcal{L}_{x}}=0,\left.\quad G\right|_{\boldsymbol{r} \in \mathcal{L}_{\Lambda}}=0 .
$$

Equation (B.6) can be easily derived from the divergence (Gauss) theorem applied to $G\left(\left|\boldsymbol{r}-\boldsymbol{r}^{\prime}\right|\right)$ inside a circle of radius $R$ centered at $\boldsymbol{r}^{\prime}$. It reduces to

$$
1=2 \pi R \frac{d G(R)}{d R}
$$

from which one obtains directly (B.6).

The Green Function can be built by using the conformal mapping defined by $w=\exp [\pi z /(2 \Lambda)]$ with $w=$ $u+i v$ and $z=x+i y(i=\sqrt{-1})$. In real variables, it is expressed as

$$
\begin{aligned}
& u=\exp [\pi x /(2 \Lambda)] \cos [\pi y /(2 \Lambda)] \\
& v=\exp [\pi x /(2 \Lambda)] \sin [\pi y /(2 \Lambda)]
\end{aligned}
$$

This conformal mapping transforms the infinite long strip $\mathcal{D}$ into the quarter of plane such that $u \geq 0$ and $v \geq 0$ (see fig. B.1). The boundary $y=0$ is mapped to the line $v=0$, $u>0$, and the boundary $y=\Lambda$ to the line $u=0, v>0$. Therefore, eqs. (B.7) map to the boundary conditions

$$
\left.\frac{\partial G}{\partial v}\right|_{v=0, u>0}=0,\left.\quad G\right|_{u=0, v>0}=0
$$

The solution is easy to construct by the mirror reflection method, by placing two point sources at locations $\left(u^{\prime}, v^{\prime}\right)$ and $\left(u^{\prime},-v^{\prime}\right)$, and two point sinks at locations $\left(-u^{\prime}, v^{\prime}\right)$ and $\left(-u^{\prime},-v^{\prime}\right)$. One gets

$$
\begin{aligned}
& G\left(u, v, u^{\prime}, v^{\prime}\right)= \\
& \quad \frac{1}{4 \pi} \ln \frac{\left[\left(u-u^{\prime}\right)^{2}+\left(v-v^{\prime}\right)^{2}\right]\left[\left(u-u^{\prime}\right)^{2}+\left(v+v^{\prime}\right)^{2}\right]}{\left[\left(u+u^{\prime}\right)^{2}+\left(v-v^{\prime}\right)^{2}\right]\left[\left(u+u^{\prime}\right)^{2}+\left(v+v^{\prime}\right)^{2}\right]} .
\end{aligned}
$$

The solution $G\left(x, y, x^{\prime}, y^{\prime}\right)$ can be readily obtained by substitution of the relations (B.8, B.9).

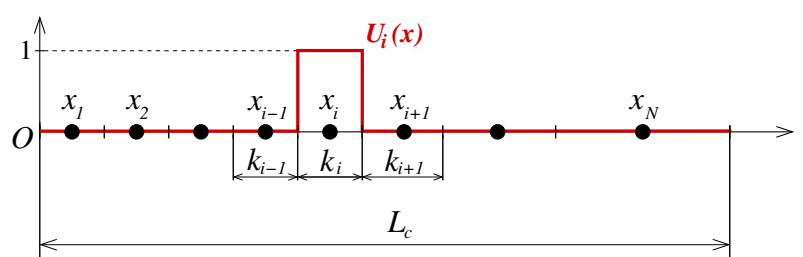

Fig. C.1: Mesh and interpolation function $U_{i}(x)$.

By using eqs. (B.5, B.2, B.4) and (B.7), eq. (B.1) in $\mathcal{D}$ reduces to

$$
\varsigma\left(\boldsymbol{r}^{\prime}\right)=\int_{\mathcal{L}_{x}}\left[\varsigma(\boldsymbol{r}) \nabla_{r} G\left(\boldsymbol{r}, \boldsymbol{r}^{\prime}\right)-G\left(\boldsymbol{r}, \boldsymbol{r}^{\prime}\right) \nabla \varsigma(\boldsymbol{r})\right] \cdot \boldsymbol{n} d l_{r}
$$

To obtain the governing equation connecting the reduced vapor concentration at the interface and the mass flux, one needs to consider the solution for $y^{\prime}=0, x^{\prime}>0$,

$$
\varsigma\left(x^{\prime}, y^{\prime}=0\right)=-\int_{0}^{\infty} G_{0}\left(x, x^{\prime}\right) j_{1}(x) d x,
$$

where

$$
\begin{aligned}
G_{0}\left(x, x^{\prime}\right)=G\left(x, y=0, x^{\prime}, y^{\prime}=0\right)= \\
\frac{1}{\pi} \ln \left|\frac{\exp \frac{\pi x}{2 \Lambda}-\exp \frac{\pi x^{\prime}}{2 \Lambda}}{\exp \frac{\pi x}{2 \Lambda}+\exp \frac{\pi x^{\prime}}{2 \Lambda}}\right| .
\end{aligned}
$$

By using the relation $\varsigma=c_{1}-1$, one obtains eq. (36).

\section{Solution method for the integro-differential equation}

The tilde denoting dimensionless quantities is omitted in this appendix for clarity.

Equation (38) is solved numerically. The unknown function $j_{1}(x)$ is interpolated in the domain $\left[0, L_{c}\right]$ with $L_{c}$ an arbitrary cut-off such that $L_{c} \gg \Lambda$. The interpolation in the domain $\left[0, L_{c}\right]$ is performed by splitting this interval into $N$ subintervals of length $k_{i}(i=1$ to $N)$, and by using the interpolation functions $U_{i}(x)=H\left(x_{i}-k_{i} / 2\right)-H\left(x_{i}+\right.$ $\left.k_{i} / 2\right)$, with $H(x)$ the Heaviside function and $x_{i}$ the center of the $i^{\text {th }}$ subinterval (see figure C.1).

The approximate expression for $j_{1}(x)$ then reads

$$
j_{1}(x)=\sum_{i=1}^{N} j^{(i)} U_{i}(x)+H\left(x-L_{c}\right) f(x) .
$$

where $f(x)$ is an asymptotic expansion of $j_{1}(x)$ at large $x$, which can be obtained a priori. Indeed, at a large $x$, the concentration field in the gas phase is such that $\left|\partial c_{1} / \partial x\right| \ll\left|\partial c_{1} / \partial y\right|$, which allows to consider a lubricationlike approximation for the diffusion equation (a sufficient condition for this approximation to be valid being $x \gg \Lambda$ ). The Laplace equation (25) thus reduces to $\partial^{2} c_{1} / \partial y^{2}=0$ 
and eq. (28) to $j_{1}=\left(c_{i, 1}-1\right) / \Lambda$. Using relation (28), one gets $\mathcal{K}_{1}=-(\Lambda+\mathcal{R}) j_{1}-1$. Combining this relation with equation (24) leads to

$$
\frac{d}{d x}\left(x^{3} \frac{d j_{1}}{d x}\right)=\frac{j_{1}}{(\Lambda+\mathcal{R})}
$$

with the boundary condition $\lim _{x \rightarrow \infty} j_{1}(x)=-1 /(\Lambda+\mathcal{R})$ that corresponds to zero curvature at infinity. The truncation of the solution for large $x$ yields

$$
f(x)=-\frac{1}{(\Lambda+\mathcal{R})}+\frac{1}{(\Lambda+\mathcal{R})^{2} x}
$$

to be used in equation C.1.

It is worthwhile to point out that the asymptotics (C.3) is different from the asymptotics (39) for the second intermediate region obtained from equation (A.12). Indeed, the model developed in Appendix A ignores the microscale effects. They however are accounted for here. An important consequence is that the numerical solution of the equation (38) can describe the whole liquid wedge, i.e. from $x=0$ to infinity. While the Kelvin effect declines outside the microregion, the interfacial resistance persists and thus contributes to the relation (C.3). One notes however that, in reality, $\Lambda \gg \mathcal{R}$ by many orders of magnitude.

There are $N$ unknowns in the expression (C.1), thus requiring $N$ equations. The discretized version of equation (38) is

$$
\begin{array}{r}
x_{i}^{3} \sum_{m=1}^{N} C_{i m} j^{(m)}-\mathcal{R} x_{i}^{3} \frac{j^{(i+1)}-j^{(i)}}{x_{i+1}-x_{i}}+\sum_{m=1}^{i-1} j^{(m)} k_{m}+j^{(i)} \frac{k_{i}}{2} \\
\left.=x_{i}^{3} D_{i} \text { for } i=1,2, \ldots N, \quad \text { (C. } 4\right)
\end{array}
$$

with $x_{N+1}=L_{c}$ and $j^{(N+1)}=-(\Lambda+\mathcal{R})^{-1}+(\Lambda+\mathcal{R})^{-2} L_{c}^{-1}$. The coefficients $C_{i m}$ and $D_{i}$ are obtained with the expression (B.14) for the Green function:

$$
\begin{aligned}
C_{i m}= & \int_{x_{m}-k_{m} / 2}^{x_{m}+k_{m} / 2} \frac{\partial G_{0}}{\partial x}\left(x_{i}, x^{\prime}\right) d x^{\prime}, \\
D_{i}= & \frac{1}{(\Lambda+\mathcal{R})} \int_{L_{c}}^{\infty} \frac{\partial G_{0}}{\partial x}\left(x_{i}, x^{\prime}\right) d x^{\prime} \\
& -\frac{1}{(\Lambda+\mathcal{R})^{2}} \int_{L_{c}}^{\infty} \frac{1}{x^{\prime}} \frac{\partial G_{0}}{\partial x}\left(x_{i}, x^{\prime}\right) d x^{\prime} .
\end{aligned}
$$

The integral in eq. (C.5) and the first integral in eq. (C.6) are computed analytically. Due to the interchangeability of $x$ and $x^{\prime}$ in the expression for $G_{0}$ (B.14), the antiderivative with respect of $x^{\prime}$ of $\partial G_{0} / \partial x$ is simply equal to $-G_{0}$. Note that the kernel of the integral (C.5) diverges when $x^{\prime}=x_{i}$, which happens when $m=i$. In this case, one can easily check that the integral has a zero Cauchy Principal Value, so $C_{i i}=0$. The last integral in eq. (C.6) is computed numerically, by adding $N_{a}$ additional nodes in the

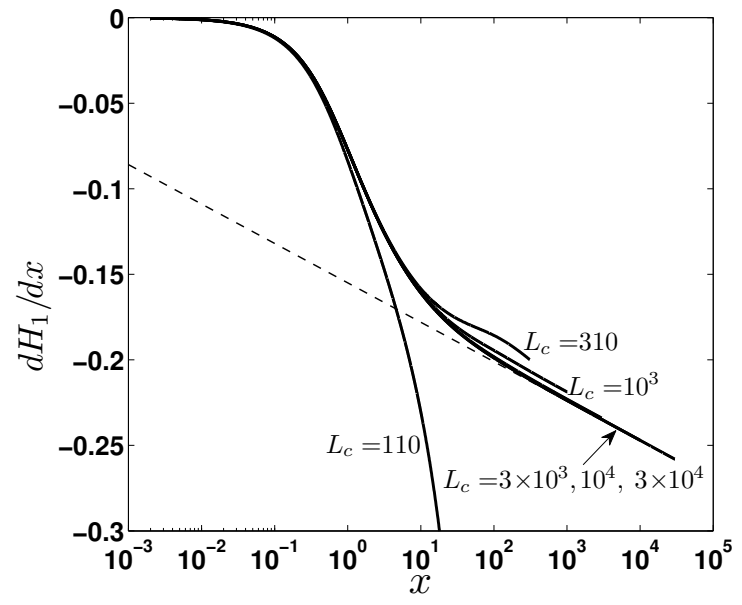

Fig. C.2: Convergence test for the numerical treatment when increasing the cut-off $L_{c}$ for $\Lambda=100$ and $\mathcal{R}=0$.

domain $\left[L_{c}, L_{\infty}\right]$, with $L_{\infty}$ such that $L_{c} \ll L_{\infty}$ :

$$
\begin{aligned}
\int_{L_{c}}^{\infty} \frac{1}{x^{\prime}} \frac{\partial G_{0}}{\partial x}\left(x_{i}, x^{\prime}\right) d x^{\prime} \simeq \int_{L_{c}}^{L_{\infty}} \frac{1}{x^{\prime}} \frac{\partial G_{0}}{\partial x}\left(x_{i}, x^{\prime}\right) d x^{\prime} \\
\\
\simeq \sum_{m=N+1}^{N+N_{a}} \frac{C_{i m}}{x_{m}} .
\end{aligned}
$$

$L_{\infty}$ is increased until convergence is achieved.

The effect of the cut-off $L_{c}$ on the slope $d H_{1} / d x$ is presented in fig. C.2. It shows a good convergence for $L_{c}>$ 3000 .

\section{Numerical integration to get $\mathcal{K}_{1}$ from $j_{1}$}

The tilde denoting dimensionless quantities is omitted in this appendix for clarity. Computing the curvature $\mathcal{K}_{1}\left(x_{i}\right)$ from eq. (37) requires numerical evaluation of an integral which involves the Green function $G_{0}\left(x_{i}, x^{\prime}\right)$. Using the discretization of $j_{1}$ given by eq. (C.1), the integral is evaluated from

$$
\begin{aligned}
& \int_{0}^{\infty} G_{0}\left(x_{i}, x^{\prime}\right) j_{1}\left(x^{\prime}\right) d x^{\prime} \simeq \\
& \sum_{m=1}^{N} j^{(m)} \int_{x_{m}-k_{m} / 2}^{x_{m}+k_{m} / 2} G_{0}\left(x_{i}, x^{\prime}\right) d x^{\prime}+\alpha \int_{L_{c}}^{\infty} \frac{G_{0}\left(x_{i}, x^{\prime}\right)}{x^{\prime p}} d x^{\prime} .
\end{aligned}
$$

The second integral in the rhs of relation (D.1) is approximated by adding $N_{a}$ additional nodes in the range $\left[L_{c}, L_{\infty}\right]$ with $L_{c} \ll L_{\infty}$, as already done in eq. (C.7):

$$
\begin{aligned}
\int_{L_{c}}^{\infty} \frac{G_{0}\left(x_{i}, x^{\prime}\right)}{x^{\prime p}} d x^{\prime} & \simeq \int_{L_{c}}^{L_{\infty}} \frac{G_{0}\left(x_{i}, x^{\prime}\right)}{x^{\prime p}} d x^{\prime} \\
& \simeq \sum_{m=N+1}^{N+N_{a}} x_{m}^{-p} \int_{x_{m}-k_{m} / 2}^{x_{m}+k_{m} / 2} G_{0}\left(x_{i}, x^{\prime}\right) d x^{\prime} .
\end{aligned}
$$


F. Doumenc et al.: Thin wedge evaporation/condensation controlled by the vapor dynamics in the atmosphere

Because of the presence of an absolute value in eq. (B.14), two cases must be considered when computing the antiderivative of $G_{0}\left(x_{i}, x^{\prime}\right)$ (defined as usually within a constant). For $x^{\prime}<x_{i}$,

$$
\begin{aligned}
& \int G_{0}\left(x_{i}, x^{\prime}\right) d x^{\prime}= \\
& \frac{\Lambda}{\pi^{2}}\left(-4 \operatorname{Li}_{2}\left\{\exp \left[\frac{\pi}{2 \Lambda}\left(x^{\prime}-x_{i}\right)\right]\right\}+\right. \\
&\left.\operatorname{Li}_{2}\left\{\exp \left[\frac{\pi}{\Lambda}\left(x^{\prime}-x_{i}\right)\right]\right\}\right),
\end{aligned}
$$

where $\mathrm{Li}_{2}$ is the dilogarithm function. For $x_{i}<x^{\prime}$,

$$
\begin{aligned}
\int G_{0}\left(x_{i}, x^{\prime}\right) d x^{\prime}= & \\
- & \frac{2 \Lambda}{\pi^{2}}\left(\operatorname{Li}_{2}\left\{1-\exp \left[\frac{\pi}{2 \Lambda}\left(x_{i}-x^{\prime}\right)\right]\right\}\right. \\
& \left.+\operatorname{Li}_{2}\left\{-\exp \left[\frac{\pi}{2 \Lambda}\left(x_{i}-x^{\prime}\right)\right]\right\}\right) \\
+ & \frac{1}{\pi}\left(x^{\prime}-x_{i}\right) \ln \left\{1-\exp \left[\frac{\pi}{2 \Lambda}\left(x_{i}-x^{\prime}\right)\right]\right\} .
\end{aligned}
$$

Notice that $G_{0}\left(x_{i}, x^{\prime}\right)$ diverges when $x^{\prime}=x_{i}$, but the integral converges.

\section{References}

1. M. Potash, P.C. Wayner, Int. J. Heat Mass Transfer 15, $1851(1972)$

2. D.M. Anderson, S.H. Davis, Phys. Fluids 7, 248 (1995)

3. V.S. Nikolayev, Phys. Fluids 22, 082105 (2010)

4. R.D. Deegan, O. Bakajin, T.F. Dupont, G. Huber, S.R. Nagel, T.A. Witten, Phys. Rev. E 62, 756 (2000)

5. Y.O. Popov, Phys. Rev. E 71, 036313 (2005)

6. H. Hu, R. Larson, J. Phys. Chem. B 106, 1334 (2002)

7. C. Poulard, G. Guéna, A.M. Cazabat, A. Boudaoud, M. Ben Amar, Langmuir 21, 8226 (2005)

8. G. Berteloot, C.T. Pham, A. Daerr, F. Lequeux, L. Limat, Europhys. Lett. 83, 14003 (2008)

9. C.T. Pham, G. Berteloot, F. Lequeux, L. Limat, Europhys. Lett. 92, 54005 (2010)

10. J. Eggers, L.M. Pismen, Phys. Fluids 22, 112101 (2010)

11. S.J.S. Morris, J. Fluid Mech. 739, 308 (2014)

12. C. Huh, L.E. Scriven, J. Colloid Interf. Sci. 35, 85 (1971)

13. L.M. Hocking, Q. J. Mechanics Appl. Math. 36, 55 (1983)

14. V. Janeček, B. Andreotti, D. Pražák, T. Bárta, V.S. Nikolayev, Phys. Rev. E 88, 060404 (2013)

15. A. Rednikov, P. Colinet, Phys. Rev. E 87, 010401 (2013)
16. J.H. Snoeijer, B. Andreotti, Annu. Rev. Fluid Mech. 45, 269 (2013)

17. S. Moosman, G.M. Homsy, J. Colloid Interface Sci. 73, $212(1980)$

18. P. Stephan, J. Hammer, Heat Mass Transfer 30, 119 (1994)

19. A.Y. Rednikov, P. Colinet, Langmuir 27, 1758 (2011)

20. V. Janeček, V.S. Nikolayev, Europhys. Lett. 100, 14003 (2012)

21. R. Raj, C. Kunkelmann, P. Stephan, J. Plawsky, J. Kim, Int. J. Heat Mass Transfer 55, 2664 (2012)

22. L. Fourgeaud, E. Ercolani, J. Duplat, P. Gully, V.S. Nikolayev, Phys. Rev. Fluids 1, 041901 (2016)

23. V. Janeček, V.S. Nikolayev, Phys. Rev. E 87, 012404 (2013)

24. Y. Tsoumpas, S. Dehaeck, M. Galvagno, A. Rednikov, H. Ottevaere, U. Thiele, P. Colinet, Langmuir 30, 11847 (2014)

25. F. Doumenc, B. Guerrier, Eur. Phys. J. Special Topics 197, 281 (2011)

26. V.V. Janeček, F. Doumenc, B. Guerrier, V.S. Nikolayev, J. Colloid Interface Sci. 460, 329 (2015)

27. C. Loussert, F. Doumenc, J.B. Salmon, V.S. Nikolayev, B. Guerrier, Langmuir 33, 14078 (2017)

28. G. Barnes, Adv. Colloid Interface Sci. 25, 89 (1986)

29. V.P. Carey, Liquid-Vapor Phase Change Phenomena (Hemisphere, Washington D.C., 1992)

30. M.A. Saxton, D. Vella, J.P. Whiteley, J.M. Oliver, J. Eng. Math. 106, 47 (2017)

31. J.H. Snoeijer, J. Eggers, Phys. Rev. E 82, 056314 (2010)

32. J. Eggers, Phys. Fluids 17, 082106 (2005)

33. A. Korn, T.M. Korn, Mathematical Handbook for Scientists and Engineers: Definitions, Theorems, and Formulas for Reference and Review (Mc Graw Hill, New York, 2000)

34. G. Grinberg, Selected Problems of the Mathematical Theory of Electric and Magnetic Effects (Izd. Akad. Nauk SSSR, Moscow-Leningrad, 1948), in Russian 